\title{
Mapping single-cell atlases throughout Metazoa unravels cell type evolution
}

3 Alexander J. Tarashansky ${ }^{1}$, Jacob M. Musser ${ }^{2}, \S$, Margarita Khariton ${ }^{1, \S}$, Pengyang Li ${ }^{1}$,

$6 \quad{ }^{1}$ Department of Bioengineering, Stanford University, Stanford, CA, USA.

7 2European Molecular Biology Laboratory, Developmental Biology Unit, Heidelberg,

8 Germany.

$9 \quad{ }^{3}$ Centre for Organismal Studies, University of Heidelberg, Heidelberg, Germany.

10 4Department of Applied Physics, Stanford University, Stanford, CA, USA.

$11{ }^{5}$ Chan Zuckerberg Biohub, San Francisco, CA, USA.

$12{ }^{6}$ Department of Developmental Biology, Stanford University School of Medicine, Stanford,

13 CA, USA.

14

15 §These authors contributed equally to this work.

16

$17{ }^{*}$ Correspondence: wangbo@stanford.edu. 


\section{Abstract}

19 Comparing single-cell transcriptomic atlases from diverse organisms can elucidate the

20 origins of cellular diversity and assist the annotation of new cell atlases. Yet,

21 comparison between distant relatives is hindered by complex gene histories and

22 diversifications in expression programs. Previously, we introduced the self-assembling

23 manifold (SAM) algorithm to robustly reconstruct manifolds from single-cell data

24 (Tarashansky et al., 2019). Here, we build on SAM to map cell atlas manifolds across

25 species. This new method, SAMap, identifies homologous cell types with shared

26 expression programs across distant species within phyla, even in complex examples

27 where homologous tissues emerge from distinct germ layers. SAMap also finds many

28 genes with more similar expression to their paralogs than their orthologs, suggesting

29 paralog substitution may be more common in evolution than previously appreciated.

30 Lastly, comparing species across animal phyla, spanning mouse to sponge, reveals

31 ancient contractile and stem cell families, which may have arisen early in animal

32 evolution. 


\section{Introduction}

34 There is much ongoing success in producing single-cell transcriptomic atlases to

35 investigate the cell type diversity within individual organisms (Regev et al., 2017). With

36 the growing diversity of cell atlases across the tree of life (Briggs et al., 2018; Cao et al.,

37 2019; Fincher et al., 2018; Hu et al., 2020; Musser et al., 2019; Plass et al., 2018; Siebert

38 et al., 2019; Wagner et al., 2018), a new frontier is emerging: the use of cross-species

39 cell type comparisons to unravel the origins of cellular diversity and uncover species-

40 specific cellular innovations (Arendt et al., 2019; Shafer, 2019). Further, these

41 comparisons promise to accelerate cell type annotation and discovery by transferring

42 knowledge from well-studied model organisms to under-characterized animals.

44 However, recent comparative single-cell analyses are mostly limited to species within the 45 same phylum (Baron et al., 2016; Geirsdottir et al., 2019; Sebé-Pedrós et al., 2018;

46 Tosches et al., 2018). Comparisons across longer evolutionary distances and across

47 phyla are challenging for two major reasons. First, gene regulatory programs diversify

48 during evolution, diminishing the similarities in cell type specific gene expression patterns.

49 Second, complex gene evolutionary history causes distantly related organisms to share

50 few one-to-one gene orthologs (Nehrt et al., 2011), which are often relied upon for

51 comparative studies (Briggs et al., 2018; Shafer, 2019). This effect is compounded by the

52 growing evidence suggesting that paralogs may be more functionally similar than

53 orthologs across species, due to differential gain (neo-functionalization), loss (non-

54 functionalization), or partitioning (sub-functionalization) events among paralogs (Nehrt et

55 al., 2011; Prince \& Pickett, 2002; Stamboulian et al., 2020; Studer \& Robinson-Rechavi, 56 2009). 
58 Here, we present the Self-Assembling Manifold mapping (SAMap) algorithm to enable

59 mapping single-cell transcriptomes between phylogenetically remote species. SAMap

60 relaxes the constraints imposed by sequence orthology, using expression similarity

61 between mapped cells to infer the relative contributions of homologous genes, which in

62 turn refines the cell type mapping. In addition, SAMap uses a graph-based data

63 integration technique to identify reciprocally connected cell types across species with

64 greater robustness than previous single-cell data integration methods (Haghverdi et al.,

65 2018; Hie et al., 2019; Polański et al., 2019; Stuart et al., 2019).

67 Using SAMap, we compared seven whole-body cell atlases from species spanning animal

68 phylogeny, which have divergent transcriptomes and complex molecular homologies

69 (Figure 1A-B and Supplementary Table 1). We began with well-characterized cell types

70 in developing frog and fish embryos. We found broad concordance between

71 transcriptomic signatures and ontogenetic relationships, which validated our mapping

72 results, yet also detected striking examples of homologous cell types emerging from

73 different germ layers. We next extended the comparison to animals from the same phylum

74 but with highly divergent body plans, using a planarian flatworm and a parasitic blood

75 fluke, and found one-to-one homologies even between cell subtypes. Comparing all

76 seven species from sponge to mouse, we identified densely interconnected cell type

77 families broadly shared across animals, including contractile and stem cells, along with

78 their respective gene expression programs. Lastly, we noticed that homologous cell types

79 often exhibit differential expression of orthologs and similar expression of paralogs, 
80 suggesting that the substitution and swapping of paralogs in cell types may be more

81 common in evolution than previously appreciated. Overall, our study represents an

82 important step towards analyzing the evolutionary origins of specialized cell types and

83 their associated gene expression programs in animals. 


\section{Results}

\section{The SAMap algorithm}

86 SAMap iterates between two modules. The first module constructs a gene-gene bipartite

87 graph with cross-species edges connecting homologous gene pairs, initially weighted by

88 protein sequence similarity (Figure $1 \mathrm{C}$ ). In the second module, SAMap uses the gene-

89 gene graph to project the two single-cell transcriptomic datasets into a joint, lower-

90 dimensional manifold representation, from which each cell's mutual cross-species

91 neighbors are linked to stitch the cell atlases together (Figure 1D). Then, using the joint

92 manifold, the expression correlations between homologous genes are computed and

93 used to reweight the edges in the gene-gene homology graph in order to relax SAMap's

94 initial dependence on sequence similarity. The new homology graph is used as input to

95 the subsequent iteration of SAMap, and the algorithm continues until convergence,

96 defined as when the cross-species mapping does not significantly change between

97 iterations (Figure 1E).

98

99 This algorithm overcomes several challenges inherent to mapping single-cell

100 transcriptomes between distantly related species. First, complex gene evolutionary

101 history often results in many-to-many homologies with convoluted functional relationships

102 (Briggs et al., 2018; Nehrt et al., 2011). SAMap accounts for this by using the full

103 homology graph to project each dataset into both its own and its partner's respective

104 principal component (PC) spaces, constructed by the SAM algorithm, which we previously

105 developed to robustly and sensitively identify cell types (Tarashansky et al., 2019). The

106 resulting within- and cross-species projections are concatenated to form the joint space. 
107 For the cross-species projections, we translate each species' features into those of its

108

109

110

111

112

113

114

115

116

117

partner, with the expression for individual genes imputed as the weighted average of their

homologs specified in the gene-gene bipartite graph. Iteratively refining the homology graph to only include positively correlated gene pairs prunes the many-to-many homologies to only include genes that are expressed in the same mapped cell types.

Second, frequent gene losses and the acquisitions of new genes result in many cell type gene expression signatures being species-specific, limiting the amount of information that is comparable across species. Restricting the analysis of each dataset to only include genes that are shared across species would result in a decreased ability to resolve cell types and subtypes with many species-specific gene signatures. SAMap solves this problem by constructing the joint space through the concatenation of within- and crossspecies projections, thus encoding all genes from both species.

Lastly, the evolution of expression programs gradually diminishes the similarity between homologous cell types. To account for this effect, SAMap links cell types across species while tolerating their differences. Cells are mapped by calculating each of their $k$ mutual nearest cross-species neighbors in the combined projection. To establish more robust mutual connectivity, we integrate information from each cell's local, within-species neighborhood (Figure 1D), overcoming the inherent stochasticity of cross-species correlations. Two cells are thus defined as mutual nearest cross-species neighbors when their respective neighborhoods have mutual connectivity. It is important to note that the magnitude of connections is not directly calculated from their expression similarity, 
130 allowing cell types with diverged expression profiles to be tightly linked if they are among each other's closest cross-species neighbors.

Paralog substitutions are prevalent between homologous cell types in frog and fish

134 We first applied SAMap to the Xenopus and zebrafish atlases, which both encompass

135 embryogenesis until early organogenesis (Briggs et al., 2018; Wagner et al., 2018).

136 Previous analysis had linked cell types between these two organisms by matching 137 ontogeny, thereby providing a reference for comparison. SAMap produced a combined 138 manifold with a high degree of cross-species alignment while maintaining high resolution 139 for distinguishing cell types in each species (Figure 2A). We measured the mapping 140 strength between cell types by calculating an alignment score (edge width in Figure 2B 141 and color map in Figure 2C), defined as the average number of mutual nearest cross142 species neighbors of each cell relative to the maximum possible number of neighbors.

144 SAMap revealed broad agreement between transcriptomic similarity and developmental 145 ontogeny, linking 26 out of 27 expected pairs based on previous annotations (Figure 2B 146 and Supplementary Table 2) (Briggs et al., 2018). The only exception is the embryonic

147 kidney (pronephric duct/mesenchyme), potentially indicating that their gene expression 148 programs have significantly diverged. In addition, SAMap succeeded in drawing parallels 149 between the development of homologous cell types and matched time points along 150 several cell lineages (Figure 2C). While the concordance was consistent across cell 151 types, we noticed that the exact progression of developmental timing can vary, suggesting 152 that SAMap can quantify heterochrony with cell type resolution. 
154 SAMap also weakly linked several closely related cell types with different ontogeny. For example, optic cells from both species are also connected to eye primordium, frog skeletal

156 muscles to fish presomitic mesoderm, and frog hindbrain to fish forebrain/midbrain.

157 Notable exceptions also included mapped secretory cell types that differ in their

158 developmental origin and even arise from different germ layers (black edges in Figure 159 2B). They are linked through a large set of genes including conserved transcription factors

160 (e.g., foxa1 (Dubaissi et al., 2014), grhl (Miles et al., 2017)) and proteins involved in 161 vesicular protein trafficking (Figure 2 - figure supplement 1). This observation supports 162 the notion that cell types may be transcriptionally and evolutionarily related despite having 163 different developmental origins (Arendt et al., 2016).

165 To benchmark SAMap performance, we used Eggnog (Huerta-Cepas et al., 2019) to 166 define one-to-one vertebrate orthologs between fish and frog and fed these gene pairs 167 as input to several broadly used single-cell data integration methods, Seurat (Stuart et 168 al., 2019), Liger (Welch et al., 2019), Harmony (Korsunsky et al., 2019), Scanorama (Hie et al., 2019), and BBKNN (Polański et al., 2019). We found that they failed to map the two 170 atlases, yielding minimal alignment between them (Figure 2D and Figure 2 - figure 171 supplement 2). We also compared the results when restricting SAMap to using the one172 to-one orthologs instead of the full homology graph. Even when removing the many-to173 many gene homologies and the iterative refinement of the homology graph, we identified 174 similar, albeit weaker, cell type mappings. This suggests that, at least for the frog and fish 
175 comparison, SAMap's performance is owed in large part to its robust, atlas stitching 176 approach.

178 The key benefit of using the full homology graph is to enable the systematic identification 179 of gene paralogs that exhibit greater similarity in expression across species than their 180 corresponding orthologs. These events are expected to arise as the result of gene 181 duplications followed by diversification of the resulting in-paralogs (Studer \& Robinson182 Rechavi, 2009). In addition, genetic compensation by transcriptional adaptation, where 183 loss-of-function mutations are balanced by upregulation of related genes with similar 184 sequences (El-Brolosy et al., 2019), could also result in this signature. In total, SAMap 185 selected 8,286 vertebrate orthologs and 7,093 eukaryotic paralogs, as enumerated by 186 Eggnog, for manifold alignment. Among these, 565 genes have markedly higher 187 expression correlations (correlation difference $>0.3$ ) with their paralogs than their 188 orthologs (Figure 2E and Figure 2 - figure supplement 3 ), and 209 genes have 189 orthologs that are either completely absent or lowly-expressed with no cell-type specificity 190 (Supplementary Table 3), suggesting that they may have lost their functional roles at 191 some point and were compensated for by their paralogs. We term these events as 192 "paralog substitutions". SAMap linked an additional 297 homologous pairs not previously 193 annotated by orthology or paralogy, but which exhibited sequence similarity and high 194 expression correlations (>0.5 Pearson correlation). These likely represent unannotated 195 orthologs/paralogs or isofunctional, distantly related homologs (Gabaldón \& Koonin, 196 2013). These results illustrate the potential of SAMap in leveraging single-cell gene 197 expression data for pruning the networks of homologous genes to identify evolutionary 
substitution of paralogs and, more generally, identify non-orthologous gene pairs that may perform similar functions in the cell types within which they are expressed.

Homologous cell types between two flatworm species with divergent body plans

To test if we can identify homologous cell types in animals with radically different body

203 plans, we mapped the cell atlases of two flatworms, the planarian Schmidtea

204 mediterranea (Fincher et al., 2018), and the trematode Schistosoma mansoni, which we

205 collected recently (Li et al., 2020). They represent two distant lineages within the same

206 phylum but have remarkably distinct body plans and autecology (Laumer et al., 2015;

207 Littlewood \& Waeschenbach, 2015). While planarians live in freshwater and are known

208 for their ability to regenerate (Reddien, 2018), schistosomes live as parasites in humans.

209 The degree to which cell types are conserved between them is unresolved, given the vast

210 phenotypic differences caused by the transition from free-living to parasitic habits

211 (Laumer et al., 2015).

212

213 SAMap revealed broad cell type homology between schistosomes and planarians. The

214 schistosome had cells mapped to the planarian stem cells, called neoblasts, as well as

215 most of the differentiated tissues: neural, muscle, intestine, epidermis, parenchymal,

216 protonephridia, and cathepsin ${ }^{+}$cells, the latter of which consists of cryptic cell types that,

217 until now, have only been found in planarians (Fincher et al., 2018) (Figure 3A). These

218 mappings are supported by both known cell type specific marker genes and numerous

219 homologous transcriptional regulators (Figure 3B and Figure 3 - figure supplement 1). 
221 We next determined if cell type homologies exist at the subtype level. For this, we compared the neoblasts, as planarian neoblasts are known to comprise populations of

223 pluripotent cells and tissue-specific progenitors (Fincher et al., 2018; Zeng et al., 2018).

224 By mapping the schistosome neoblasts to a planarian neoblast atlas (Zeng et al., 2018), we found that the schistosome has a population of neoblasts ( $\varepsilon$-cells (Wang et al., 2018))

226 that cluster with the planarian's pluripotent neoblasts, both expressing a common set of 227 TFs (e.g., soxp2, unc4, pax6a, gcm1) (Figure 3C-D). The $\varepsilon$-cells are closely associated 228 with juvenile development and lost in adult schistosomes (Wang et al., 2018), indicating 229 pluripotent stem cells may be a transient population restricted to their early developmental

230 stages. This is consistent with the fact that, whereas schistosomes can heal wounds, they 231 have limited regenerative ability (Wendt \& Collins, 2016). SAMap also linked other 232 schistosome neoblast populations with planarian progenitors, including two populations 233 of schistosome neoblasts (denoted as $\mu$ (Tarashansky et al., 2019) and $\mu$ ') to planarian 234 muscle progenitors, all of which express myoD, a canonical master regulator of 235 myogenesis (Scimone et al., 2017). These likely represent early and late muscle 236 progenitors, respectively, as $\mu$-cells do not yet express differentiated muscle markers 237 such as troponin, whereas $\mu$ '-cells do (Figure 3 - figure supplement 2).

\section{Cell type families spanning the animal tree of life}

240 To compare cell types across broader taxonomic scales, we extended our analysis to 241 include juvenile freshwater sponge (Spongilla lacustris) (Musser et al., 2019), adult Hydra

242 (Hydra vulgaris) (Siebert et al., 2019), and mouse (Mus musculus) embryogenesis 243 (Pijuan-Sala et al., 2019) atlases. In total, SAMap linked 1,051 cross-species pairs of cell 
244 types, defined by the annotations used in each respective study. $95 \%$ of the cell type 245 pairs are supported by at least 40 enriched gene pairs, and $87 \%$ are supported by more

246 than 100 gene pairs, indicating that SAMap does not spuriously connect cell types with

247 limited overlap in expression profiles (Figure 4A).

248

249 We next extended the notion of cell type pairs to cell type trios, as mapped cell types gain 250 additional support if they share transitive relationships to other cell types through

251 independent mappings, forming cell type triangles among species. The transitivity of a 252 cell type pair (edge) or a cell type (node) can be quantified as the fraction of triads to 253 which they belong that form triangles (Figure 4B). The majority (81\%) of cell type pairs 254 have non-zero transitivity independent of alignment score and the number of enriched 255 gene pairs (Figure 4 - figure supplement 1-2). Cell type pairs with fewer than 40 256 enriched gene pairs tend to have lower (<0.4) transitivity (Figure 4 - figure supplement 257 2). In addition, $16 \%$ of mapped cell type pairs have zero edge transitivity but non-zero 258 node transitivity, meaning that at least one of the cell types connects to only a single 259 member of an interconnected cell type group (Figure 4C). Such edges may be of lower 260 confidence as they should connect to other members of the same group and are thus 261 excluded from downstream analysis.

262

263 Among the interconnected groups of cell types, we identified families of contractile cells 264 and neural cells (Figure 4D). Both cell type families are highly transitive compared to the 265 overall graph transitivity (bootstrap $p$-value $<1 \times 10^{-5}$ ), meaning that their constituent cell 266 types have more transitive edges within the group than outside the group (Figure 4E). In 
267 addition, the dense, many-to-many connections within the contractile and neural families

268 are each supported by at least 40 enriched gene pairs (Figure 4F). Consistent with the

269 nerve net hypothesis suggesting a unified origin of neural cell types (Tosches \& Arendt,

270 2013), the neural family includes vertebrate brain tissues, both bilaterian and cnidarian

271 neurons, cnidarian nematocytes that share the excitatory characteristics of neurons (Weir

272 et al., 2020), and Spongilla choanocytes and apopylar cells, both of which are not

273 considered as neurons but have been shown to express postsynaptic-like scaffolding

274 machinery (Musser et al., 2019; Wong et al., 2019). The contractile family includes

275 myocytes in bilaterian animals, Hydra myoepithelial cells that are known to have

276 contractile myofibrils (Buzgariu et al., 2015), and sponge pinacocytes and

277 myopeptidocytes, both of which have been implicated to play roles in contractility (Musser

278 et al., 2019; Sebé-Pedrós et al., 2018). In contrast to the families encompassing all seven

279 species, we also found a fully interconnected group that contains invertebrate pluripotent

280 stem cells, including planarian and schistosome neoblasts, Hydra interstitial cells, and

281 sponge archeocytes (Alié et al., 2015). The lack of one-to-one connections across phyla

282 is in keeping with recent hypotheses that ancestral cell types diversified into families of

283 cell types after speciation events (Arendt et al., 2016, 2019). Our findings thus suggest

284 that these cell type families diversified early in animal evolution.

285

286 Transcriptomic signatures of cell type families

287 The high interconnectedness between cell types across broad taxonomic scales suggests

288 that they should share ancestral transcriptional programs (Arendt et al., 2016; Tosches

289 et al., 2018). SAMap identified broad transcriptomic similarity between bilaterian and non- 
290 bilaterian contractile cells that extends beyond the core contractile apparatus. It links a 291 total of 23,601 gene pairs, connecting 5,471 unique genes, which are enriched in at least

292 one contractile cell type pair. Performing functional enrichment analysis on these genes, 293 we found cytoskeleton and signal transduction functions to be enriched ( $p$-value $<10^{-3}$ ) 294 based on the KOG functional classifications (Tatusov et al., 2003) assigned by Eggnog

295 (Figure 5A). These genes include orthology groups spanning diverse functional roles in 296 contractile cells, including actin regulation, cell adhesion and stability, and signaling 297 (Figure 5B and Supplementary Table 4), indicating that contractile cells were likely 298 multifunctional near the beginning of animal evolution.

300 We also identified several transcriptional regulators shared among contractile cells 301 (Figure 5B). Previously known core regulators involved in myocyte specification (Brunet 302 et al., 2016) were enriched only in bilaterian (e.g., myod, and tcf4/E12) or vertebrate 303 contractile cells (e.g., mef2). In contrast, we found homologs of Muscle Lim Protein (Csrp) 304 and Forkhead Box Group 1 (Larroux et al., 2008) enriched in contractile cells from all 305 seven species. The Fox proteins included FoxC, which is known to regulate cardiac 306 muscle identity in vertebrates (Brunet et al., 2016) and is contractile-specific in all species 307 except schistosome and Spongilla. Notably, we also identified FoxG orthologs to be 308 enriched in three of the four invertebrates (Figure 5 - figure supplement 1), suggesting 309 that FoxG may play an underappreciated role in contractile cell specification outside 310 vertebrates. 
312 For the family of invertebrate stem cells, we identified 3,343 genes that are enriched in at

313 least one cell type pair and observed significant enrichment $\left(p-v a l u e<10^{-3}\right)$ of genes

314 involved in translational regulation such as RNA processing, translation, and post-

315 translational modification (Figure 5C). These genes form 979 orthology groups, $17 \%$ of

316 which are enriched in all cell types of this family (Supplementary Table 4). Importantly,

317 other stem cell populations in Hydra and planarian lineage-restricted neoblasts have

318 significantly reduced expression of these genes (Figure 5D). These results suggest that

319 SAMap identified a large, deeply conserved gene module specifically associated with

320 multipotency.

\section{Discussion}

323 Cell types evolve as their gene expression programs change either as integrated units or

324 via evolutionary splitting that results in separate derived programs. While this notion of 325 coupled cellular and molecular evolution has gained significant traction in the past years, 326 systematically comparing cell type-specific gene expression programs across species 327 has remained a challenging problem. Here, we map single-cell atlases between 328 evolutionarily distant species in a manner that accounts for the complexity of gene 329 evolution. SAMap aligns cell atlases in two mutually reinforcing directions, mapping both 330 the genes and the cells, with each feeding back into the other. This method allows us to 331 identify one-to-one cell type concordance between animals in the same phylum, whereas 332 between phyla, we observe interconnected cell types forming distinct families. These

333 findings support the notion that cell types evolve via hierarchical diversification (Arendt et 334 al., 2019), resulting in cell type families composed of evolutionarily related cell types 
sharing a regulatory gene expression program that originated in their common ancestor.

336 One-to-one cell type homologies should exist only if no further cell type diversification has

337 occurred since the speciation.

In parallel, SAMap systematically identifies instances where paralogs exhibit greater

340 expression similarity than orthologs across species. Paralog substitution likely occurs due

341 to differential loss or retention of cell type-specific expression patterns of genes that were

342 duplicated in the common ancestor (Studer \& Robinson-Rechavi, 2009). Alternatively,

343 paralog substitutions could arise due to compensating upregulation of paralogs following

344 a loss-of-function mutation acquired by an ortholog (El-Brolosy et al., 2019). While the

345 analysis presented here focuses on comparisons between two species, incorporating

346 multiple species into a single analysis that also accounts for their phylogenetic

347 relatedness could enable determining the specific order of paralog substitutions,

348 associated cell type diversification events, and the mechanism by which they arose.

349 However, this would require cell atlases that consistently sample key branching points

350 along the tree of life. Nevertheless, identifying lineage-specific paralog substitution

351 signatures should be accessible in extensively studied vertebrate single-cell atlases, as

352 the vertebrate clade is where existing data and knowledge are most concentrated.

354 Besides applications in evolutionary biology, we anticipate SAMap can catalyze the 355 annotation of new cell atlases from non-model organisms, which often represents a 356 substantial bottleneck requiring extensive manual curation and prior knowledge. Its ability 357 to use the existing atlases to inform the annotation of cell types in related species will 
358 keep improving as more datasets become available to better sample the diversity of cell

359 types. Moreover, our approach allows leveraging existing and forthcoming single-cell

360 gene expression data to predict functionally similar gene homologs, which can serve as

361 guideposts for mechanistic molecular studies.

362 


\section{Materials and Methods}

\section{Data and Code Availability}

- The source code for SAMap is publicly available at Github (https://github.com/atarashansky/SAMap), along with the code to perform the analysis and generate the figures.

- The datasets analyzed in this study are detailed in Supplementary Table 1 with their accessions, and annotations provided.

373 The SAMap algorithm contains three major steps: preprocessing, mutual nearest

374 neighborhood alignment, and gene-gene correlation initialization. The latter two are

375 repeated for three iterations, by default, to balance alignment performance and 376 computational runtime. SAMap runs up to one hour on an average desktop computer for 377200,000 total cells.

\section{Preprocessing.}

380 1.1. Generate gene homology graph via reciprocal BLAST.

381 We first construct a gene-gene bipartite graph between two species by performing 382 reciprocal BLAST of their respective transcriptomes using tblastx, or proteomes using 383 blastp. tblastn and blastx are used for BLAST between proteome and transcriptome. 384 When a pair of genes share multiple High Scoring Pairs (HSPs), which are local regions 
385 of matching sequences, we use the HSP with the highest bit score to measure homology.

386 Only pairs with E-value $<10^{-6}$ are included in the graph.

388 Here we define similarity using BLAST, though SAMap is compatible with other protein homology detection methods (e.g. HMMER (Eddy, 2008)) or orthology inference tools (e.g. OrthoClust (Yan et al., 2014) and Eggnog (Huerta-Cepas et al., 2019)). While each

of these methods has known strengths and limitations, BLAST is chosen for its broad usage, technical convenience, and compatibility with low-quality transcriptomes.

We encode the BLAST results into two triangular adjacency matrices, $A$ and $B$, each containing bit scores in one BLAST direction. We combine $A$ and $B$ to form a gene-gene adjacency matrix $G$. After symmetrizing $G$, we remove edges that only appear in one

397 direction: $G=\operatorname{Recip}\left(\frac{1}{2}\left[(A+B)+(A+B)^{T}\right]\right) \in \mathfrak{R}^{m_{1}+m_{2} \times m_{1}+m_{2}}$, where $\operatorname{Recip}$ only keeps 398 reciprocal edges, and $m_{1}$ and $m_{2}$ are the number of genes of the two species, respectively. To filter out relatively weak homologies, we also remove edges where $G_{a b}<$ gene and transformed by a hyperbolic tangent function to increase discriminatory power between low and high edge weights, $\widehat{G}_{a b}=0.5+0.5 \tanh \left(10 G_{a b} / \max _{b}\left(G_{a b}\right)-5\right)$.

405 The scRNAseq datasets are normalized such that each cell has a total number of raw 406 counts equal to the median size of single-cell libraries. Gene expressions are then log407 normalized with the addition of a pseudocount of 1. Genes expressed (i.e., $\log _{2}(D+1)>$ 
1) in greater than $96 \%$ of cells are filtered out. SAM is run using the following parameters:

$411 N_{1}$ and $N_{2}$, which are directed adjacency matrices that encode $k$-nearest neighbor graphs

412 for the two datasets, respectively.

414 SAM only includes the top 3,000 genes ranked by SAM weights and the first 150 principal

415 components (PCs) in the default mode to reduce computational complexity. However, 416 downstream mapping requires PC loadings for all genes. Thus, in the final iteration of 417 SAM, we run PCA on all genes and take the top 300 PCs. This step generates a loading 418 matrix for each species $i, L_{i} \in \mathfrak{R}^{300 \times m_{i}}$.

\section{Mutual nearest neighborhood alignment.}

2.1. Transform feature spaces between species.

422 For the gene expression matrices $Z_{i} \in \Re^{n_{i} \times m_{i}}$, where $n$ and $m$ are the number of cells 423 and genes respectively, we first zero the expression of genes that do not have an edge

424 in $\hat{G}$ and standardize the expression matrices such that each gene has zero mean and 425 unit variance, yielding $\tilde{Z}_{i}$. $\hat{G}$ represents a bipartite graph in the form of $\hat{G}=$ $426\left[\begin{array}{cc}0_{m_{1}, m_{1}} & H \in \mathfrak{R}^{m_{1} \times m_{2}} \\ H^{T} \in \mathfrak{R}^{m_{2} \times m_{1}} & 0_{m_{2}, m_{2}}\end{array}\right]$, where $0_{m, m}$ is $m \times m$ zero matrix and $H$ is the biadjacency

427 matrix. Letting $H_{1}=H$ and $H_{2}=H^{T}$ encoding directed edges from species 1 to 2 and 2

428 to 1 , respectively, we normalize the biadjacency matrix $H_{i}$ such that each row sums to 1:

$429 \widehat{H}_{i}=\operatorname{SumNorm}\left(H_{i}\right) \in \mathfrak{R}^{m_{i} \times m_{j}}$, where the SumNorm function normalizes the rows to sum 
430 to 1 . The feature spaces can be transformed between the two species via weighted

431 averaging of gene expression, $\tilde{Z}_{i j}=\tilde{Z}_{i} \widehat{H}_{i}$.

432

433 2.2. Project single-cell gene expressions into a joint PC space.

434 We project the expression data from two species into a joint PC space (Barkas et al., 435 2019), $P_{i}=\tilde{Z}_{i} L_{i}{ }^{T}$ and $P_{i j}=\tilde{Z}_{i j} L_{j}{ }^{T}$. We then horizontally concatenate the principal 436 components $P_{i}$ and $P_{i j}$ to form $\hat{P}_{i} \in \Re^{n_{i} \times 600}$.

2.3. Calculate k-nearest cross-species neighbors for all cells.

439 Using the joint PCs, $\hat{P}_{i}$, we identify for each cell the $k$-nearest neighbors in the other 440 dataset using cosine similarity ( $k=20$ by default). Neighbors are identified using the 441 hnswlib library, a fast approximate nearest-neighbor search algorithm (Malkov \& 442 Yashunin, 2020). This outputs two directed biadjacency matrices $C_{i} \in \mathfrak{R}^{n_{i} \times n_{j}}$ for $(i, j)=$ $443(1,2)$ or $(2,1)$ with edge weights equal to the cosine similarity between the PCs.

445 2.4. Apply the graph-coarsening mapping kernel to identify cross-species mutual nearest 446 neighborhoods.

447 To increase the stringency and confidence of mapping, we only rely on cells that are 448 mutual nearest cross-species neighbors, which are typically defined as two cells 449 reciprocally connected to one another (Haghverdi et al., 2018). However, due to the noise 450 in cell-cell correlations and stochasticity in the kNN algorithms, cross-species neighbors 451 are often randomly assigned from a pool of cells that appear equally similar, decreasing 452 the likelihood of mutual connectivity between individual cells even if they have similar 
453 expression profiles. To overcome this limitation, we integrate information from each cell's

454 local neighborhood to establish more robust mutual connectivity between cells across

455 species. Two cells are thus defined as mutual nearest cross-species neighbors when their

456 respective neighborhoods have mutual connectivity.

458 Specifically, the nearest neighbor graphs $N_{i}$ calculated in step 1.2 are used to calculate 459 the neighbors of cells $t_{i}$ hops away along outgoing edges: $\bar{N}_{i}=N_{i}{ }_{i}$, where $\bar{N}_{i}$ are 460 adjacency matrices that contain the number of paths connecting two cells $t_{i}$ hops away,

461 for $i=1$ or $2 . t_{i}$ determines the length-scale over which we integrate incoming edges for 462 species $i$. Its default value is 2 if the dataset size is less than 20,000 cells and 3 otherwise. 463 However, cells within tight clusters may have spurious edges connecting to other parts of 464 the manifold only a few hops away. To avoid integrating neighborhood information outside 465 this local structure, we use the Leiden algorithm (Traag et al., 2019) to cluster the graph 466 and identify a local neighborhood size for each cell (the resolution parameter is set to 3 467 by default). If cell $a$ belongs to cluster $c_{a}$, then its neighborhood size is $l_{a}=\left|c_{a}\right|$. For each 468 row $a$ in $\bar{N}_{i}$ we only keep the $l_{a}$ geodesically closest cells, letting the pruned graph be 469 denoted as $\widehat{N}_{i}$.

471 Edges outgoing from cell $a_{i}$ in species $i$ are encoded in the corresponding row in the 472 adjacency matrix: $C_{i, a_{i}}$. We compute the fraction of the outgoing edges from each cell that 473 target the local neighborhood of a cell in the other species: $\tilde{C}_{i, a_{i} b_{j}}=\sum_{c \in X_{j, b_{j}}} C_{i, a_{i} c}$, where $474 X_{j, b_{j}}$ is the set of cells in the neighborhood of cell $b_{j}$ in species $j$ and $\tilde{C}_{i, a_{i} b_{j}}$ is the fraction 
475 of outgoing edges from cell $a_{i}$ in species $i$ targeting the neighborhood of cell $b_{j}$ in species

$476 j$.

477

478 To reduce the density of $\tilde{C}_{i}$ so as to satisfy computational memory constraints, we remove

479 edges with weight less than 0.1. Finally, we apply the mutual nearest neighborhood

480 criterion by taking the element-wise, geometric mean of the two directed bipartite graphs:

$481 \tilde{C}=\sqrt{\tilde{C}_{1} \circ \tilde{C}_{2}}$. This operation ensures that only bidirectional edges are preserved, as small

482 edge weights in either direction results in small geometric means.

483

484 2.5. Assign the k-nearest cross-species neighborhoods for each cell and update edge 485 weights in the gene homology graph.

486 Given the mutual nearest neighborhoods $\tilde{C} \in \mathfrak{R}^{n_{1} \times n_{2}}$, we select the $k$ nearest 487 neighborhoods for each cell in both directions to update the directed biadjacency matrices

2.6. Stitch the manifolds.

491 We use $C_{1}$ and $C_{2}$ to combine the manifolds $N_{1}$ and $N_{2}$ into a unified graph. We first weight 492 the edges in $N_{1}$ and $N_{2}$ to account for the number of shared cross-species neighbors by 493 computing the one-mode projections of $C_{1}$ and $C_{2}$. In addition, for cells with strong cross494 species alignment, we attenuate the weight of their within-species edges. For cells with 495 little to no cross-species alignment, their within-species are kept the same to ensure that 496 the local topological information around cells with no alignment is preserved. 
498 Specifically, we use $N_{1}$ and $N_{2}$ to mask the edges in the one-mode projections, $\widetilde{N}_{1}=$ $U\left(N_{1}\right) \circ\left(\operatorname{Norm}\left(C_{1}\right) \operatorname{Norm}\left(C_{2}\right)\right)$ and $\widetilde{N}_{2}=U\left(N_{2}\right) \circ\left(\operatorname{Norm}\left(C_{2}\right) \operatorname{Norm}\left(C_{1}\right)\right)$, where $U(E)$ sets

500 all edge weights in graph $E$ to 1 and Norm normalizes the outgoing edges from each cell

501 to sum to 1 . The minimum edge weight is set to be 0.3 to ensure that neighbors in the 502 original manifolds with no shared cross-species neighbors still retain connectivity: $\widetilde{N}_{1, i j}=$ $503 \min \left(0.3, \widetilde{N}_{1, i j}\right)$ and $\widetilde{N}_{2, i j}=\min \left(0.3, \widetilde{N}_{2, i j}\right)$ for all edges $(i, j)$. We then scale the within504 species edges from cell $i$ by the total weight of its cross-species edges: $\widetilde{N}_{1, i}=(1-$ $\left.505 \frac{1}{k} \sum_{j=1}^{n_{2}} C_{1, i j}\right) \widetilde{N}_{1, i}$ and $\widetilde{N}_{2, i}=\left(1-\frac{1}{k} \sum_{j=1}^{n_{1}} C_{2, i j}\right) \widetilde{N}_{2, i}$. Finally, the within- and cross-species 506 graphs are stitched together to form the combined nearest neighbor graph $N: N=\left[\widetilde{N}_{1} \oplus\right.$ $\left.507 C_{1}\right] \oplus\left[C_{2} \oplus \widetilde{N}_{2}\right]$. The overall alignment score between species 1 and 2 is defined as $S=$ $508 \frac{1}{n_{1}+n_{2}}\left(\sum_{i=1}^{n_{1}} \sum_{j=1}^{n_{2}} C_{1, i j}+\sum_{i=1}^{n_{2}} \sum_{j=1}^{n_{1}} C_{2, i j}\right)$.

\section{Gene-gene correlation initialization.}

511 3.1. Update edge weights in the gene-gene bipartite graph with expression correlations.

512 To compute correlations between gene pairs, we first transfer expressions from one 513 species to the other: $\bar{Z}_{i, n_{i} m_{j}}=C_{i, n_{i}} Z_{j, m_{j}}$, where $\bar{Z}_{i, n_{i} m_{j}}$ is the imputed expressions of gene $514 m_{j}$ from species $j$ for cell $n_{i}$ in species $i$, and $C_{i, n_{i}}$ is row $n_{i}$ of the biadjacency matrix

515 encoding the cross-species neighbors of cell $n_{i}$ in species $i$, all for $(i, j)=(1,2)$ and $(2,1)$.

516 We similarly use the manifolds constructed by SAM to smooth the within-species gene 517 expressions using kNN averaging: $\bar{Z}_{j, m_{j}}=N_{j, m_{j}} Z_{j, m_{j}}$, where $N_{j}$ is the nearest-neighbor 518 graph for species $j$. We then concatenate the within- and cross-species gene expressions such that the expression of gene $m_{j}$ from species $j$ in both species is $\bar{Z}_{m_{j}}=\bar{Z}_{i, m_{j}} \oplus \bar{Z}_{j, m_{j}}$. 
521 For all gene pairs in the unpruned homology graph generated in step 1.1., $\widehat{G}$, we compute

522 their correlations, $\widehat{G}_{a b}:=\theta(0) \operatorname{Corr}\left(\bar{Z}_{a}, \bar{Z}_{b}\right)$, where $\theta(0)$ is a Heaviside step function

523 centered at 0 to set negative correlations to zero. We then use the expression correlations

524 to update the corresponding edge weights in $\hat{G}$, which are again normalized through

$525 \widehat{G}_{a b}=0.5+0.5 \tanh \left(10 \widehat{G}_{a b} / \max _{b}\left(\widehat{G}_{a b}\right)-5\right)$.

\section{Annotation of cell atlases}

528 To annotate the primary zebrafish and Xenopus cell types, the cell subtype annotations 529 provided by the original publications (Briggs et al., 2018; Wagner et al., 2018) are

530 coarsened using a combination of the manual matching and developmental hierarchies.

531 For example, as "heart - mature", "heart - hoxd9a", "heart", and "heart field" in zebrafish

532 are all manually matched to "cardiac mesoderm" in Xenopus, we label these cells as

533 "heart". In cases where the matching is insufficient to coarsen the annotations, we use

534 the provided developmental trees to name a group of terminal cell subtypes by their

535 common ontogenic ancestor. The annotations provided by their respective studies were

536 used to label the cells in the Spongilla, Hydra, and mouse atlases. To annotate the

537 schistosome cells, we used known marker genes to annotate the main schistosome tissue

538 types (Li et al., 2020). Annotations for all single cells in all datasets are provided in

\section{Supplementary Table 1.}

\section{Visualization}

542 The combined manifold $N$ is embedded into 2D projections using UMAP implemented in 
543 the scanpy package (Wolf et al., 2018) by scanpy.tl.umap with the parameter min_dist = package.html) in $\mathrm{R}$ is used to generate the sankey plots. Edge thickness corresponds to

546 the alignment score between mapped cell types. The alignment score between cell types

$547 a$ and $b$ is defined as $s_{a b}=\frac{1}{\left|c_{a}\right|+\left|c_{b}\right|}\left(\sum_{i \in c_{a}} \sum_{j \in c_{b}} C_{1, i j}+\sum_{i \in c_{b}} \sum_{j \in c_{a}} C_{2, i j}\right)$, where $c_{a}$ and $c_{b}$

548 are the set of cells in cell types $a$ and $b$, respectively. Cell type pairs with alignment score

549 less than $z$ are filtered out. By default, $z$ is set to be 0.1 . Cell types that did not cluster 550 properly in their respective manifolds were omitted from the sankey plot. In the zebrafish-

551 Xenopus comparison, we excluded heart, germline, and olfactory placode cells from both

552 species because they did not cluster in the Xenopus atlas. Similarly, the iridoblast,

553 epiphysis, nanog+, apoptotic-like, and forerunner cells were excluded because they did 554 not cluster in the zebrafish atlas.

556 The network graphs in Figure 4D are generated using the networkx package 557 (https://networkx.github.io) in python. To focus on densely connected cell type groups, 558 we filter out cell type pairs with alignment score less than 0.05 .

\section{Identification of gene pairs that drive cell type mappings}

561 We define $g_{1}$ and $g_{2}$ to contain SAMap-linked genes from species 1 and 2, respectively.

562 Note that a gene may appear multiple times as SAMap allows for one-to-many homology.

563 Let $X_{a_{1} b_{2}}$ denote the set of all cells with cross species edges between cell types $a_{1}$ and $564 b_{2}$. We calculate the average standardized expression of all cells from species $i$ that are 565 in $X_{a_{1} b_{2}}: \quad Y_{i, g_{i}}=\frac{1}{\left|\left\{x, x \in X_{a_{1} b_{2}}\right\}\right|} \sum_{x \in X_{a_{1} b_{2}}} \tilde{Z}_{i, x, g_{i}}$, where $\tilde{Z}_{i, x, g_{i}} \in \mathfrak{R}^{\left|g_{i}\right|}$ is the standardized 
expression of genes $g_{i}$ in cell $x$. The correlation between $Y_{1, g_{1}}$ and $Y_{2, g_{2}}$ can be written as

567

568

569

570

571

572

573

574 575 of each gene in each cell type is calculated using the Wilcoxon rank-sum test implemented in the scanpy function scanpy.tl.rank_genes_groups.

$\operatorname{Corr}\left(Y_{1, g_{1}}, Y_{2, g_{2}}\right)=\sum_{j=1}^{\left|g_{1}\right|} S\left(Y_{1, g_{1}}\right)_{j} \circ S\left(Y_{2, g_{2}}\right)_{j}$, where $S(Z)$ standardizes vector $Z$ to have zero mean and unit variance. We use the summand to identify gene pairs that contribute most positively to the correlation. We assign each gene pair a score: $h_{g}=T\left(S\left(Y_{1, g_{1}}\right)\right) \circ$ $T\left(S\left(Y_{2, g_{2}}\right)\right.$ ), where $T(Z)$ sets negative values in vector $Z$ to zero in order to ignore lowlyexpressed genes. To be inclusive, we begin with the top 1,000 gene pairs according to $h_{g}$ and filter out gene pairs in which one or both of the genes are not differentially expressed in their respective cell types ( $p$-value $>10^{-2}$ ), have less than 0.2 SAM weight, or are expressed in fewer than $5 \%$ of the cells in the cluster. The differential expression

\section{8}

579

580

581

582

583

584

585

586

587

\section{Orthology group assignment}

We used the Eggnog mapper (v5.0) (Huerta-Cepas et al., 2019) to assign each gene to an orthology group with default parameters. For the zebrafish-to-Xenopus mapping, genes are considered paralogs if they map to the same eukaryotic orthology group and orthologs if they map to the same vertebrate orthology group. For the pan-species analysis, we group genes from all species with overlapping orthology assignments. In Figure 5B, each column corresponds to one of these groups. As each group may contain multiple genes from each species, we present the expression of the gene with the highest enrichment score per species. All gene names and corresponding orthology groups are reported in Supplementary Table 4. 


\section{Phylogenetic reconstruction of gene trees}

590 We generate gene trees to validate the identity of genes involved in putative examples of

591 paralog substitution and of Fox and Csrp transcriptional regulators that are identified as

592 enriched in contractile cells. For this, we first gather protein sequences from potential

593 homologs using the eggnog version 5.0 orthology database (Huerta-Cepas et al., 2019).

594 For the Fox and Csrp phylogenies, we include all Fox clade I (Larroux et al., 2008) and

595 Csrp/Crip homologs, respectively, from the seven species included in our study.

597 Alignment of protein sequences is performed with Clustal Omega version 1.2.4 using 598 default settings as implemented on the EMBL EBI web services platform (Madeira et al., 599 2019). Maximum likelihood tree reconstruction is performed using IQ-TREE version

6001.6 .12 (Nguyen et al., 2015) with the ModelFinder Plus option (Kalyaanamoorthy et al., 601 2017). For the Csrp tree, we perform 1,000 nonparametric bootstrap replicates to assess 602 node support. For Fox, we utilize the ultrafast bootstrap support option with 1,000 603 replicates. For each gene tree we choose the model that minimizes the Bayesian 604 Information Criterion (BIC) score in ModelFinder. This results in selection of the following 605 models: DCMut+R4 (Csrp) and VT+F+R5 (Fox). The final consensus trees are visualized 606 and rendered using the ete3 v3.1.1 python toolkit (Huerta-Cepas et al., 2016) and the 607 Interactive Tree of Life v4 (Letunic \& Bork, 2019).

608

609 KOG functional annotation and enrichment analysis

610 Using the eggnog mapper, KOG functional annotations are transferred to individual 611 transcripts from their assigned orthology group. For enrichment analysis, all genes 
612 enriched in the set of cell type pairs of interest are lumped to form the target set for each

613 species. For example, the target set for Spongilla archaeocytes used in Figure $\mathbf{5 C}$ is

614 composed of all genes enriched between Spongilla archaeocytes and other invertebrate

615 stem cells. Note that this set includes genes from other species that are linked by SAMap

616 to the Spongilla archeocyte genes. We include genes from other species in the target set

617 to account for differences in KOG functional annotation coverage between species. As

618 such, the annotated transcripts from all 7 species are combined to form the background

619 set. We used a hypergeometric statistical test (Eden et al., 2009) to measure the

620 enrichment of the KOG terms in the target genes compared to the background genes.

622 Mapping zebrafish and xenopus atlases using existing methods

623 For benchmarking, we used vertebrate orthologs as determined by Eggnog as input to

624 Harmony (Korsunsky et al., 2019), Liger (Welch et al., 2019), Seurat (Stuart et al., 2019),

625 Scanorama (Hie et al., 2019), BBKNN (Polański et al., 2019), which are all run with default

626 parameters. One-to-one orthologs were selected from one-to-many and many-to-many

627 orthologs by using the bipartite maximum weight matching algorithm implemented in

628 networkx. When using the one-to-one orthologs as input for SAMap, we ran for only one

629 iteration. The resulting integrated lower-dimensional coordinates (PCs for Seurat,

630 Harmony, and Scanorama and non-negative matrix factorization coordinates for Liger)

631 and stitched graph (BBKNN and SAMap) were all projected into 2D with UMAP (Figure

6322 - figure supplement 2A). The integrated coordinates are used to generate a nearest

633 neighbor graph using the correlation distance metric, which is then used to compute the 
634 alignment scores in Figure 2 - figure supplement 2B. The alignment scores for SAMap

635 and BBKNN are directly computed from their combined graphs.

637 In-situ hybridization in schistosomes

638 S. mansoni (strain: NMRI) juveniles are retrieved from infected female Swiss Webster 639 mice (NR-21963) at $\sim 3$ weeks post-infection by hepatic portal vein perfusion using $37^{\circ} \mathrm{C}$

640 DMEM supplemented with $5 \%$ heat inactivated FBS. The infected mice are provided by

641 the NIAID Schistosomiasis Resource Center for distribution through BEI Resources, NIH-

642 NIAID Contract HHSN272201000005I. In adherence to the Animal Welfare Act and the

643 Public Health Service Policy on Humane Care and Use of Laboratory Animals, all

644 experiments with and care of mice are performed in accordance with protocols approved

645 by the Institutional Animal Care and Use Committees (IACUC) of Stanford University

646 (protocol approval number 30366). In situ hybridization experiments are performed as

647 described previously (Tarashansky et al., 2019), using riboprobes synthesized from gene

648 fragments cloned with the listed primers: collagen (Smp_170340):

649 GGTGAAGAGGCTGTTGTGG, ACGATCCCCTTTCACTCCTG; $\quad$ tropomyosin

650 (Smp_031770): AAGCTGAAGTCGCCTCACTA, CATATGCCTCTTCACGCTGG;

651 troponin (Smp_018250): $\quad$ CGTAAACCTGGTCAGAAGCG,

652 ATCCTTTTCCTCCAGAGCGT; myosin regulatory light chain (Smp_132670):

653 GAGACAGCGAGTAGTGGAGG, TGCCTTCTTTGATTGGAGCT; wnt (Smp_156540):

654 TGTGGTGATGAAGATGGCAG, CCACGGCCACAACACATATT; frizzled

655 (Smp_174350): CGAACAGGCGCATGACAATA, TGCTAGTCCTGTTGTCGTGT. 


\section{Acknowledgments}

657 We thank D. Wagner and C. Juliano for sharing the data and essential discussions. We

658 also thank S. Granick, L. Luo, and J. Kebschull for their critical reading of the manuscript.

659 AJT is a Bio-X Stanford Interdisciplinary Graduate Fellow. This work is supported by a

660 Beckman Young Investigator Award and an NIH grant (1R35GM138061) to BW. 


\section{References}

662

663

664

665

666

667

668

669

670

671

672

673

674

675

676

677

678

679

680

681

682

683

684

685

686

687

688

689

690

691

692

693

694

695

696

697

698

699

700

701

702

703

704

705

706

707

708

Alié, A., Hayashi, T., Sugimura, I., Manuel, M., Sugano, W., Mano, A., Satoh, N., Agata, K., \& Funayama, N. (2015). The ancestral gene repertoire of animal stem cells. Proceedings of the National Academy of Sciences, 112(51), E7093-E7100. https://doi.org/10.1073/pnas.1514789112

Arendt, D., Bertucci, P. Y., Achim, K., \& Musser, J. M. (2019). Evolution of neuronal types and families. Current Opinion in Neurobiology, 56, 144-152. https://doi.org/10.1016/j.conb.2019.01.022

Arendt, D., Musser, J. M., Baker, C. V. H., Bergman, A., Cepko, C., Erwin, D. H., Pavlicev, M., Schlosser, G., Widder, S., Laubichler, M. D., \& Wagner, G. P. (2016). The origin and evolution of cell types. Nature Reviews Genetics, 17(12), 744-757. https://doi.org/10.1038/nrg.2016.127

Barkas, N., Petukhov, V., Nikolaeva, D., Lozinsky, Y., Demharter, S., Khodosevich, K., \& Kharchenko, P. V. (2019). Joint analysis of heterogeneous single-cell RNA-seq dataset collections. Nature Methods, 16(8), 695-698. https://doi.org/10.1038/s41592-019-0466-z

Baron, M., Veres, A., Wolock, S. L., Faust, A. L., Gaujoux, R., Vetere, A., Ryu, J. H., Wagner, B. K., Shen-Orr, S. S., Klein, A. M., Melton, D. A., \& Yanai, I. (2016). A single-cell transcriptomic map of the human and mouse pancreas reveals inter- and intra-cell population structure. Cell Systems, 3(4), 346-360.e4. https://doi.org/10.1016/j.cels.2016.08.011

Briggs, J. A., Weinreb, C., Wagner, D. E., Megason, S., Peshkin, L., Kirschner, M. W., \& Klein, A. M. (2018). The dynamics of gene expression in vertebrate embryogenesis at single-cell resolution. Science, 360(6392), eaar5780. https://doi.org/10.1126/science.aar5780

Brunet, T., Fischer, A. H., Steinmetz, P. R., Lauri, A., Bertucci, P., \& Arendt, D. (2016). The evolutionary origin of bilaterian smooth and striated myocytes. eLife, 5, e19607. https://doi.org/10.7554/eLife.19607

Buzgariu, W., Al Haddad, S., Tomczyk, S., Wenger, Y., \& Galliot, B. (2015). Multi-functionality and plasticity characterize epithelial cells in Hydra. Tissue Barriers, 3(4), e1068908. https://doi.org/10.1080/21688370.2015.1068908

Cao, C., Lemaire, L. A., Wang, W., Yoon, P. H., Choi, Y. A., Parsons, L. R., Matese, J. C., Wang, W., Levine, M., \& Chen, K. (2019). Comprehensive single-cell transcriptome lineages of a proto-vertebrate. Nature, 571(7765), 349-354. https://doi.org/10.1038/s41586-0191385-y

Dubaissi, E., Rousseau, K., Lea, R., Soto, X., Nardeosingh, S., Schweickert, A., Amaya, E., Thornton, D. J., \& Papalopulu, N. (2014). A secretory cell type develops alongside multiciliated cells, ionocytes and goblet cells, and provides a protective, anti-infective function in the frog embryonic mucociliary epidermis. Development, 141(7), 1514-1525. https://doi.org/10.1242/dev.102426

Eddy, S. R. (2008). A probabilistic model of local sequence alignment that simplifies statistical significance estimation. PLoS Computational Biology, 4(5), e1000069. https://doi.org/10.1371/journal.pcbi.1000069

Eden, E., Navon, R., Steinfeld, I., Lipson, D., \& Yakhini, Z. (2009). GOrilla: A tool for discovery and visualization of enriched GO terms in ranked gene lists. BMC Bioinformatics, 10(1), 48. https://doi.org/10.1186/1471-2105-10-48

El-Brolosy, M. A., Kontarakis, Z., Rossi, A., Kuenne, C., Günther, S., Fukuda, N., Kikhi, K., Boezio, G. L. M., Takacs, C. M., Lai, S.-L., Fukuda, R., Gerri, C., Giraldez, A. J., \& Stainier, D. Y. R. (2019). Genetic compensation triggered by mutant mRNA degradation. Nature, 568(7751), 193-197. https://doi.org/10.1038/s41586-019-1064-z 
709

710

711

712

713

714

715

716

717

718

719

720

721

722

723

724

725

726

727

728

729

730

731

732

733

734

735

736

737

738

739

740

741

742

743

744

745

746

747

748

749

750

751

752

753

754

755

756

757

758
Fincher, C. T., Wurtzel, O., de Hoog, T., Kravarik, K. M., \& Reddien, P. W. (2018). Cell type transcriptome atlas for the planarian Schmidtea mediterranea. Science, 360(6391), eaaq1736. https://doi.org/10.1126/science.aaq1736

Gabaldón, T., \& Koonin, E. V. (2013). Functional and evolutionary implications of gene orthology. Nature Reviews Genetics, 14(5), 360-366. https://doi.org/10.1038/nrg3456

Geirsdottir, L., David, E., Keren-Shaul, H., Weiner, A., Bohlen, S. C., Neuber, J., Balic, A., Giladi, A., Sheban, F., Dutertre, C.-A., Pfeifle, C., Peri, F., Raffo-Romero, A., Vizioli, J., Matiasek, K., Scheiwe, C., Meckel, S., Mätz-Rensing, K., van der Meer, F., ... Prinz, M. (2019). Cross-Species Single-Cell Analysis Reveals Divergence of the Primate Microglia Program. Cell, 179(7), 1609-1622.e16. https://doi.org/10.1016/j.cell.2019.11.010

Haghverdi, L., Lun, A. T. L., Morgan, M. D., \& Marioni, J. C. (2018). Batch effects in single-cell RNA-sequencing data are corrected by matching mutual nearest neighbors. Nature Biotechnology, 36(5), 421-427. https://doi.org/10.1038/nbt.4091

Hie, B., Bryson, B., \& Berger, B. (2019). Efficient integration of heterogeneous single-cell transcriptomes using Scanorama. Nature Biotechnology, 37(6), 685-691. https://doi.org/10.1038/s41587-019-0113-3

Hu, M., Zheng, X., Fan, C.-M., \& Zheng, Y. (2020). Lineage dynamics of the endosymbiotic cell type in the soft coral Xenia. Nature, 582(7813), 534-538. https://doi.org/10.1038/s41586020-2385-7

Huerta-Cepas, J., Serra, F., \& Bork, P. (2016). ETE 3: Reconstruction, Analysis, and Visualization of Phylogenomic Data. Molecular Biology and Evolution, 33(6), 1635-1638. https://doi.org/10.1093/molbev/msw046

Huerta-Cepas, J., Szklarczyk, D., Heller, D., Hernández-Plaza, A., Forslund, S. K., Cook, H., Mende, D. R., Letunic, I., Rattei, T., Jensen, L. J., von Mering, C., \& Bork, P. (2019). eggNOG 5.0: A hierarchical, functionally and phylogenetically annotated orthology resource based on 5090 organisms and 2502 viruses. Nucleic Acids Research, 47(D1), D309-D314. https://doi.org/10.1093/nar/gky1085

Kalyaanamoorthy, S., Minh, B. Q., Wong, T. K. F., von Haeseler, A., \& Jermiin, L. S. (2017). ModelFinder: Fast model selection for accurate phylogenetic estimates. Nature Methods, 14(6), 587-589. https://doi.org/10.1038/nmeth.4285

Korsunsky, I., Millard, N., Fan, J., Slowikowski, K., Zhang, F., Wei, K., Baglaenko, Y., Brenner, M., Loh, P., \& Raychaudhuri, S. (2019). Fast, sensitive and accurate integration of singlecell data with Harmony. Nature Methods, 16(12), 1289-1296.

https://doi.org/10.1038/s41592-019-0619-0

Larroux, C., Luke, G. N., Koopman, P., Rokhsar, D. S., Shimeld, S. M., \& Degnan, B. M. (2008). Genesis and Expansion of Metazoan Transcription Factor Gene Classes. Molecular Biology and Evolution, 25(5), 980-996. https://doi.org/10.1093/molbev/msn047

Laumer, C. E., Hejnol, A., \& Giribet, G. (2015). Nuclear genomic signals of the 'microturbellarian' roots of platyhelminth evolutionary innovation. eLife, 4, e05503. https://doi.org/10.7554/eLife.05503

Letunic, I., \& Bork, P. (2019). Interactive Tree Of Life (iTOL) v4: Recent updates and new developments. Nucleic Acids Research, 47(W1), W256-W259. https://doi.org/10.1093/nar/gkz239

Li, P., Sarfati, D. N., Xue, Y., Yu, X., Tarashansky, A. J., Quake, S. R., \& Wang, B. (2020). Single-cell analysis of Schistosoma mansoni reveals a conserved genetic program controlling germline stem cell fate [Preprint]. https://doi.org/10.1101/2020.07.06.190033

Littlewood, D. T. J., \& Waeschenbach, A. (2015). Evolution: A Turn Up for the Worms. Current Biology, 25(11), R457-R460. https://doi.org/10.1016/j.cub.2015.04.012

Madeira, F., Park, Y. mi, Lee, J., Buso, N., Gur, T., Madhusoodanan, N., Basutkar, P., Tivey, A. R. N., Potter, S. C., Finn, R. D., \& Lopez, R. (2019). The EMBL-EBI search and sequence 
802

803

804

805

806

807

808

809

analysis tools APIs in 2019. Nucleic Acids Research, 47(W1), W636-W641. https://doi.org/10.1093/nar/gkz268

Malkov, Y. A., \& Yashunin, D. A. (2020). Efficient and robust approximate nearest neighbor search using hierarchical navigable small world graphs. IEEE Transactions on Pattern Analysis and Machine Intelligence, 42(4), 824-836. https://doi.org/10.1109/TPAMI.2018.2889473

Miles, L. B., Darido, C., Kaslin, J., Heath, J. K., Jane, S. M., \& Dworkin, S. (2017). Misexpression of grainyhead-like transcription factors in zebrafish leads to defects in enveloping layer (EVL) integrity, cellular morphogenesis and axial extension. Scientific Reports, 7(1), 17607. https://doi.org/10.1038/s41598-017-17898-7

Musser, J. M., Schippers, K. J., Nickel, M., Mizzon, G., Kohn, A. B., Pape, C., Hammel, J. U., Wolf, F., Liang, C., Hernández-Plaza, A., Achim, K., Schieber, N. L., Francis, W. R., Vargas R., S., Kling, S., Renkert, M., Feuda, R., Gaspar, I., Burkhardt, P., ... Arendt, D. (2019). Profiling cellular diversity in sponges informs animal cell type and nervous system evolution [Preprint]. https://doi.org/10.1101/758276

Nehrt, N. L., Clark, W. T., Radivojac, P., \& Hahn, M. W. (2011). Testing the ortholog conjecture with comparative functional genomic data from mammals. PLoS Computational Biology, 7(6), e1002073. https://doi.org/10.1371/journal.pcbi.1002073

Nguyen, L.-T., Schmidt, H. A., von Haeseler, A., \& Minh, B. Q. (2015). IQ-TREE: A fast and effective stochastic algorithm for estimating maximum-likelihood phylogenies. Molecular Biology and Evolution, 32(1), 268-274. https://doi.org/10.1093/molbev/msu300

Pijuan-Sala, B., Griffiths, J. A., Guibentif, C., Hiscock, T. W., Jawaid, W., Calero-Nieto, F. J., Mulas, C., Ibarra-Soria, X., Tyser, R. C. V., Ho, D. L. L., Reik, W., Srinivas, S., Simons, B. D., Nichols, J., Marioni, J. C., \& Göttgens, B. (2019). A single-cell molecular map of mouse gastrulation and early organogenesis. Nature, 566(7745), 490-495. https://doi.org/10.1038/s41586-019-0933-9

Plass, M., Solana, J., Wolf, F. A., Ayoub, S., Misios, A., Glažar, P., Obermayer, B., Theis, F. J., Kocks, C., \& Rajewsky, N. (2018). Cell type atlas and lineage tree of a whole complex animal by single-cell transcriptomics. Science, 360(6391), eaaq1723. https://doi.org/10.1126/science.aaq1723

Polański, K., Young, M. D., Miao, Z., Meyer, K. B., Teichmann, S. A., \& Park, J.-E. (2019). BBKNN: Fast batch alignment of single cell transcriptomes. Bioinformatics, btz625. https://doi.org/10.1093/bioinformatics/btz625

Prince, V. E., \& Pickett, F. B. (2002). Splitting pairs: The diverging fates of duplicated genes. Nature Reviews Genetics, 3(11), 827-837. https://doi.org/10.1038/nrg928

Reddien, P. W. (2018). The Cellular and Molecular Basis for Planarian Regeneration. Cell, 175(2), 327-345. https://doi.org/10.1016/j.cell.2018.09.021

Regev, A., Teichmann, S. A., Lander, E. S., Amit, I., Benoist, C., Birney, E., Bodenmiller, B., Campbell, P., Carninci, P., Clatworthy, M., Clevers, H., Deplancke, B., Dunham, I., Eberwine, J., Eils, R., Enard, W., Farmer, A., Fugger, L., Göttgens, B., ... Human Cell Atlas Meeting Participants. (2017). The Human Cell Atlas. eLife, 6, e27041. https://doi.org/10.7554/eLife.27041

Scimone, M. L., Cote, L. E., \& Reddien, P. W. (2017). Orthogonal muscle fibres have different instructive roles in planarian regeneration. Nature, 551(7682), 623-628. https://doi.org/10.1038/nature24660

Sebé-Pedrós, A., Chomsky, E., Pang, K., Lara-Astiaso, D., Gaiti, F., Mukamel, Z., Amit, I., Hejnol, A., Degnan, B. M., \& Tanay, A. (2018). Early metazoan cell type diversity and the evolution of multicellular gene regulation. Nature Ecology \& Evolution, 2(7), 1176-1188. https://doi.org/10.1038/s41559-018-0575-6

Shafer, M. E. R. (2019). Cross-Species Analysis of Single-Cell Transcriptomic Data. Frontiers in Cell and Developmental Biology, 7, 175. https://doi.org/10.3389/fcell.2019.00175 
810

811

812

813

814

815

816

817

818

819

820

821

822

823

824

825

826

827

828

829

830

831

832

833

834

835

836

837

838

839

840

841

842

843

844

845

846

847

848

849

850

851

852

853

854

855

856

857

858

859

Siebert, S., Farrell, J. A., Cazet, J. F., Abeykoon, Y., Primack, A. S., Schnitzler, C. E., \& Juliano, C. E. (2019). Stem cell differentiation trajectories in Hydra resolved at single-cell resolution. Science, 365(6451), eaav9314. https://doi.org/10.1126/science.aav9314

Stamboulian, M., Guerrero, R. F., Hahn, M. W., \& Radivojac, P. (2020). The ortholog conjecture revisited: The value of orthologs and paralogs in function prediction. Bioinformatics, 36(Supplement_1), i219-i226. https://doi.org/10.1093/bioinformatics/btaa468

Stuart, T., Butler, A., Hoffman, P., Hafemeister, C., Papalexi, E., Mauck, W. M., Hao, Y., Stoeckius, M., Smibert, P., \& Satija, R. (2019). Comprehensive Integration of Single-Cell Data. Cell, 177(7), 1888-1902.e21. https://doi.org/10.1016/j.cell.2019.05.031

Studer, R. A., \& Robinson-Rechavi, M. (2009). How confident can we be that orthologs are similar, but paralogs differ? Trends in Genetics, 25(5), 210-216. https://doi.org/10.1016/j.tig.2009.03.004

Tarashansky, A. J., Xue, Y., Li, P., Quake, S. R., \& Wang, B. (2019). Self-assembling manifolds in single-cell RNA sequencing data. eLife, 8, e48994. https://doi.org/10.7554/eLife.48994

Tatusov, R. L., Fedorova, N. D., Jackson, J. D., Jacobs, A. R., Kiryutin, B., Koonin, E. V., Krylov, D. M., Mazumder, R., Mekhedov, S. L., Nikolskaya, A. N., Rao, B. S., Smirnov, S., Sverdlov, A. V., Vasudevan, S., Wolf, Y. I., Yin, J. J., \& Natale, D. A. (2003). The COG database: An updated version includes eukaryotes. BMC Bioinformatics, 14.

Tosches, M. A., \& Arendt, D. (2013). The bilaterian forebrain: An evolutionary chimaera. Current Opinion in Neurobiology, 23(6), 1080-1089. https://doi.org/10.1016/j.conb.2013.09.005

Tosches, M. A., Yamawaki, T. M., Naumann, R. K., Jacobi, A. A., Tushev, G., \& Laurent, G. (2018). Evolution of pallium, hippocampus, and cortical cell types revealed by single-cell transcriptomics in reptiles. Science, 360(6391), 881-888. https://doi.org/10.1126/science.aar4237

Traag, V. A., Waltman, L., \& van Eck, N. J. (2019). From Louvain to Leiden: Guaranteeing wellconnected communities. Scientific Reports, 9(1), 5233. https://doi.org/10.1038/s41598-01941695-z

Wagner, D. E., Weinreb, C., Collins, Z. M., Briggs, J. A., Megason, S., \& Klein, A. M. (2018). Single-cell mapping of gene expression landscapes and lineage in the zebrafish embryo. Science, 360(6392), 981-987.

Wang, B., Lee, J., Li, P., Saberi, A., Yang, H., Liu, C., Zhao, M., \& Newmark, P. A. (2018). Stem cell heterogeneity drives the parasitic life cycle of Schistosoma mansoni. eLife, 7, e35449. https://doi.org/10.7554/eLife.35449

Weir, K., Dupre, C., van Giesen, L., Lee, A. S.-Y., \& Bellono, N. W. (2020). A molecular filter for the cnidarian stinging response. eLife, 9, e57578. https://doi.org/10.7554/eLife.57578

Welch, J. D., Kozareva, V., Ferreira, A., Vanderburg, C., Martin, C., \& Macosko, E. Z. (2019). Single-Cell Multi-omic Integration Compares and Contrasts Features of Brain Cell Identity. Cell, 177(7), 1873-1887.e17. https://doi.org/10.1016/j.cell.2019.05.006

Wendt, G. R., \& Collins, J. J. (2016). Schistosomiasis as a disease of stem cells. Current Opinion in Genetics \& Development, 40, 95-102. https://doi.org/10.1016/j.gde.2016.06.010

Wendt, G. R., Collins, J. N., Pei, J., Pearson, M. S., Bennett, H. M., Loukas, A., Berriman, M., Grishin, N. V., \& Collins, J. J. (2018). Flatworm-specific transcriptional regulators promote the specification of tegumental progenitors in Schistosoma mansoni. eLife, 7, e33221. https://doi.org/10.7554/eLife.33221

Wolf, F. A., Angerer, P., \& Theis, F. J. (2018). SCANPY: Large-scale single-cell gene expression data analysis. Genome Biology, 19(1), 15. https://doi.org/10.1186/s13059-0171382-0

Wong, E., Mölter, J., Anggono, V., Degnan, S. M., \& Degnan, B. M. (2019). Co-expression of synaptic genes in the sponge Amphimedon queenslandica uncovers ancient neural submodules. Scientific Reports, 9(1), 15781. https://doi.org/10.1038/s41598-019-51282-x 

An orthology-based network framework for clustering data across multiple species. Genome Biology, 15(8), R100. https://doi.org/10.1186/gb-2014-15-8-r100

Zeng, A., Li, H., Guo, L., Gao, X., McKinney, S., Wang, Y., Yu, Z., Park, J., Semerad, C., Ross, E., Cheng, L.-C., Davies, E., Lei, K., Wang, W., Perera, A., Hall, K., Peak, A., Box, A., \& Sánchez Alvarado, A. (2018). Prospectively Isolated Tetraspanin+ Neoblasts Are Adult Pluripotent Stem Cells Underlying Planaria Regeneration. Cell, 173(7), 1593-1608.e20. https://doi.org/10.1016/j.cell.2018.05.006 

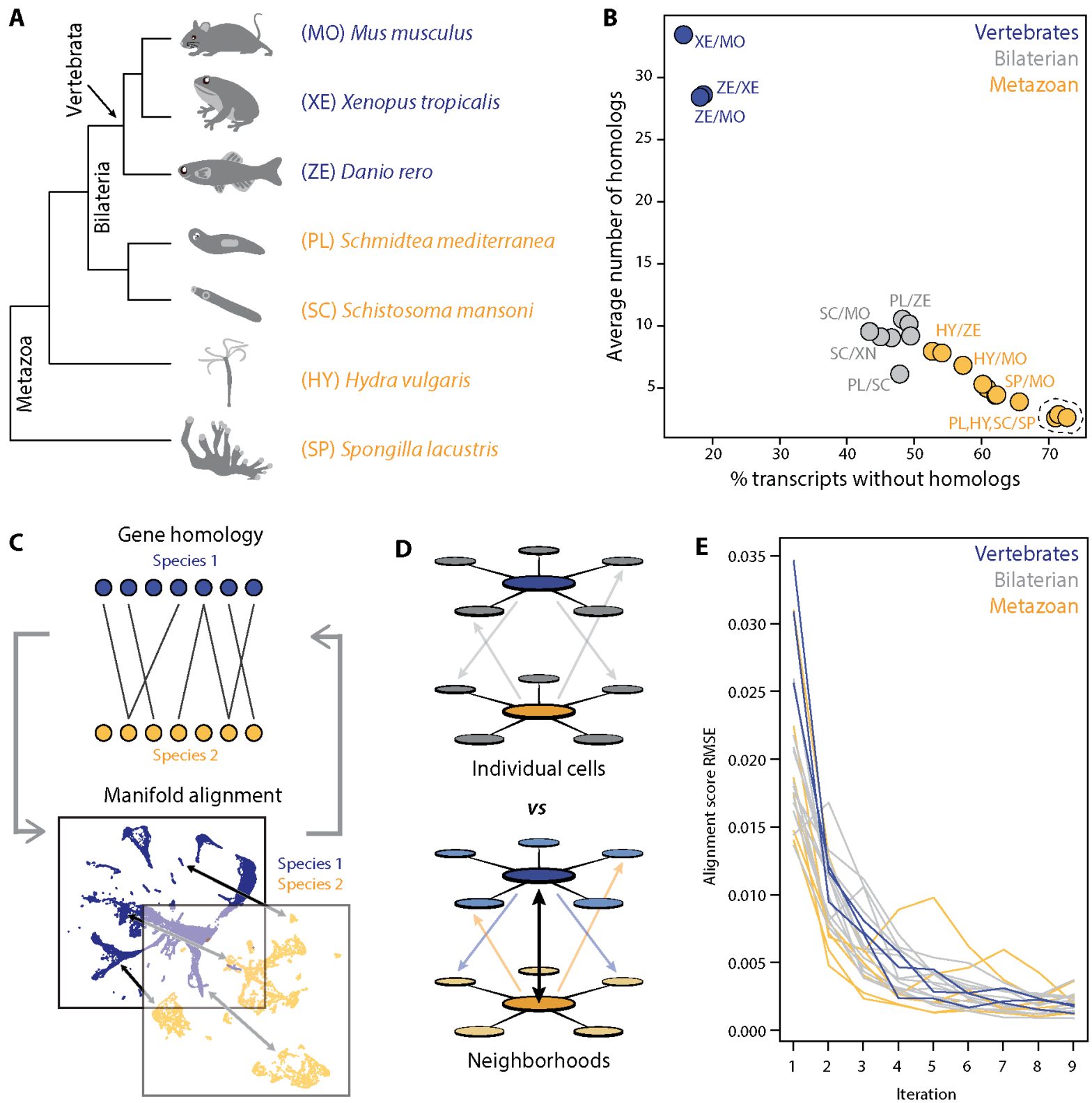

D

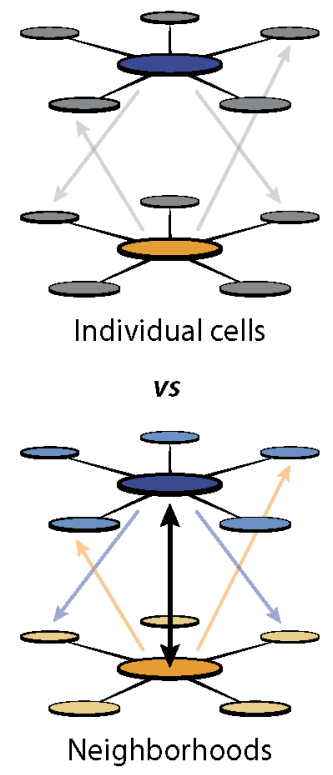

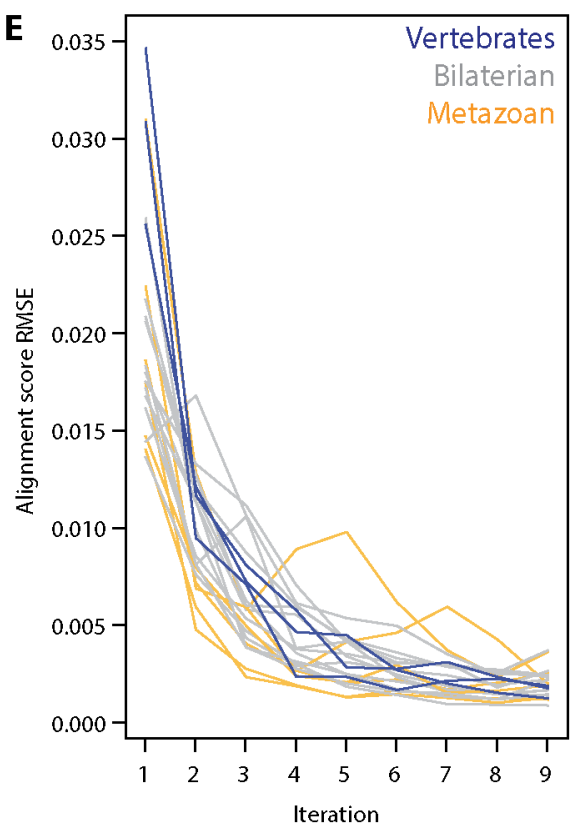

869

870 Figure 1: SAMap addresses challenges in mapping cell atlases of distantly related

871 species. (A) Schematic showing the phylogenetic relationships among 7 species

872 analyzed. (B) Challenges in mapping single-cell transcriptomes. Gene duplications cause

873 large numbers of homologs per gene, determined by reciprocal BLAST (cut-off: e-value $874<10^{-6}$ ), and frequent gene losses and the acquisition of new genes results in large 
875 fractions of transcriptomes lacking homology, which limits the amount of information

876 comparable across species. (C) SAMap workflow. Homologous gene pairs initially

877 weighted by protein sequence similarity are used to align the manifolds, low dimensional

878 representations of the cell atlases. Gene-gene correlations calculated from the aligned

879 manifolds are used to update the edge weights in the bipartite graph, which are then used

880 to improve manifold alignment. (D) Mutual nearest neighborhoods improve the detection

881 of cross-species mutual nearest neighbors by connecting cells that target one other's

882 within-species neighborhoods. (E) Convergence of SAMap is evaluated by the root mean

883 square error (RMSE) of the alignment scores between mapped clusters in adjacent

884 iterations for all 21 pairwise comparisons of the 7 species. 
bioRxiv preprint doi: https://doi.org/10.1101/2020.09.28 317784; this version posted March 19, 2021. The copyright holder for this preprint (which was not certified by peer review) is the author/funder, who has granted bioRxiv a license to display the preprint in perpetuity. It is made available under aCC-BY-NC-ND 4.0 International license.
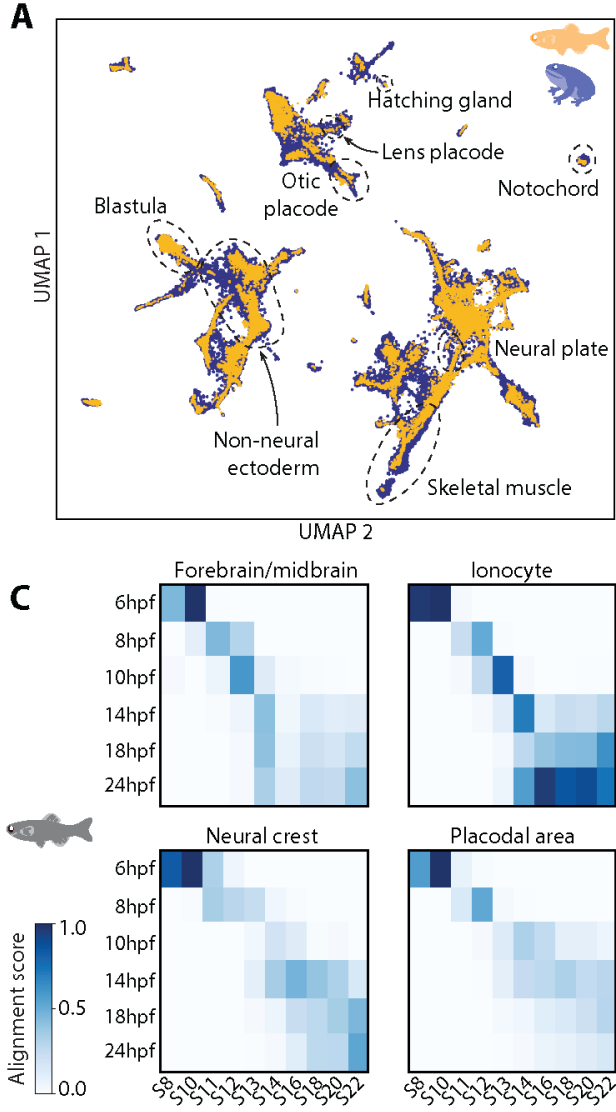

D
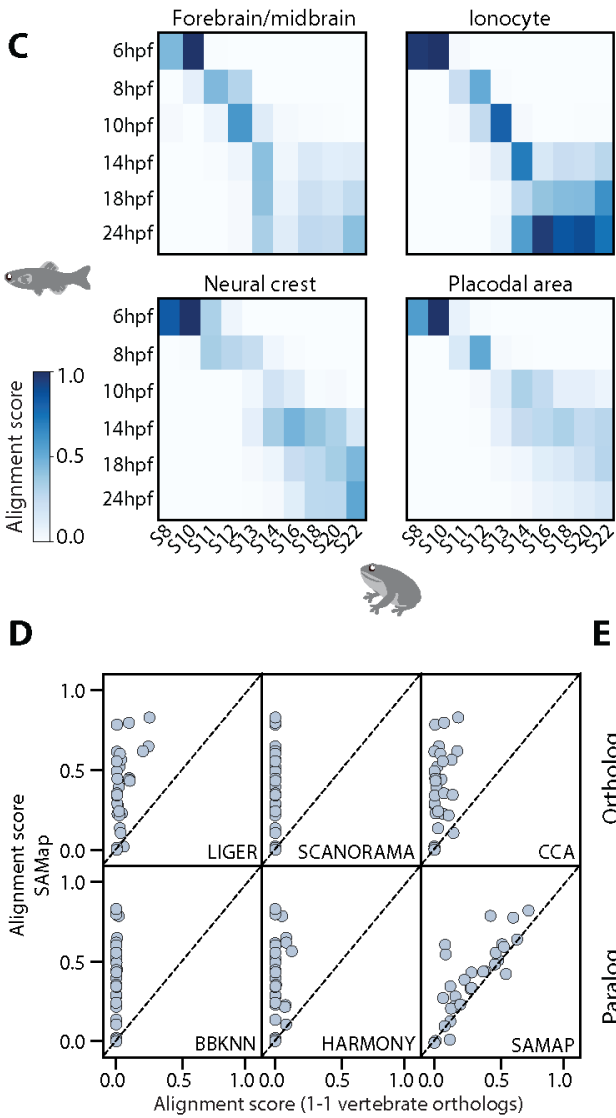

B

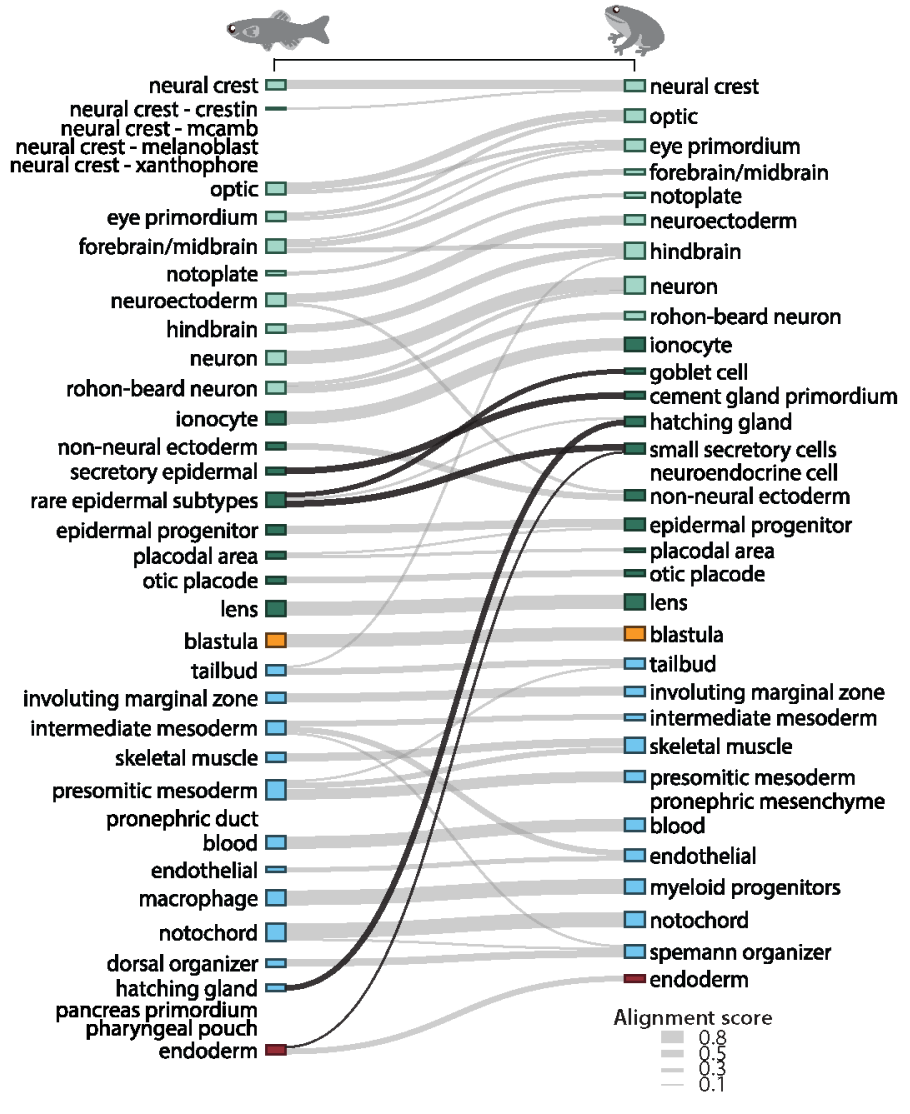

Neuroectoderm Non-neuroectoderm Mesoderm Endoderm Pluripotent

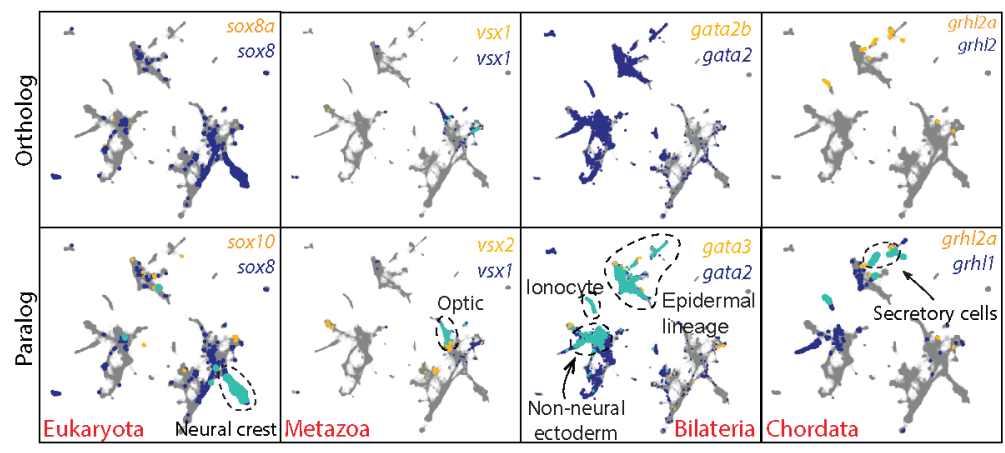

Figure 2: SAMap successfully maps $D$. rerio and $X$. tropicalis atlases and reveals 
892 Heatmaps of alignment scores between developmental time points for ionocyte, 893 forebrain/midbrain, placodal, and neural crest lineages. X-axis: zebrafish. Y-axis:

894 Xenopus. (D) SAMap alignment scores compared to those of benchmarking methods 895 using one-to-one vertebrate orthologs as input. Each dot represents a cell type pair 896 supported by ontogeny annotations. (E) Expression of orthologous (left) and paralogous

897 (right) gene pairs overlaid on the combined UMAP projection. Expressing cells are color898 coded by species, with those that are connected across species colored cyan. Cells with 899 no expression are shown in gray. More examples are provided in Figure 2 - figure 900 supplement 3.

901 

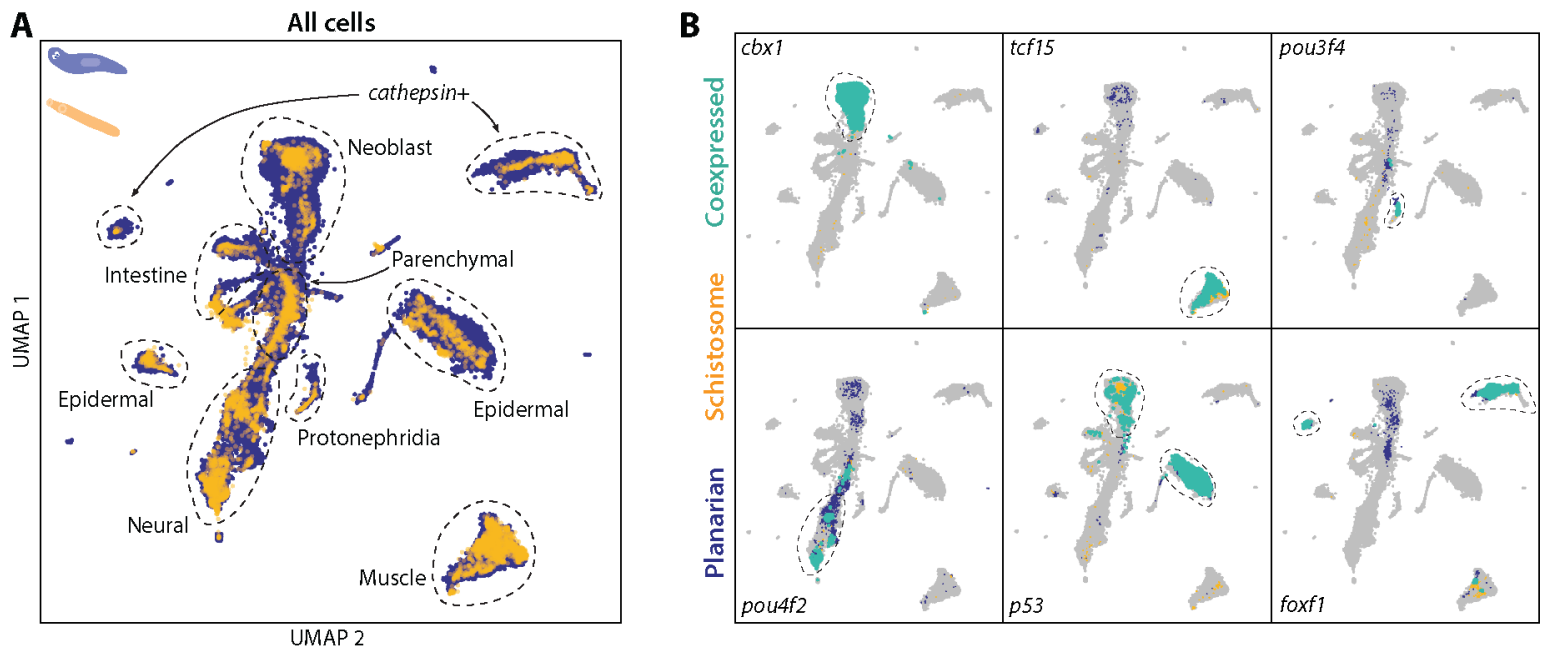

C

D
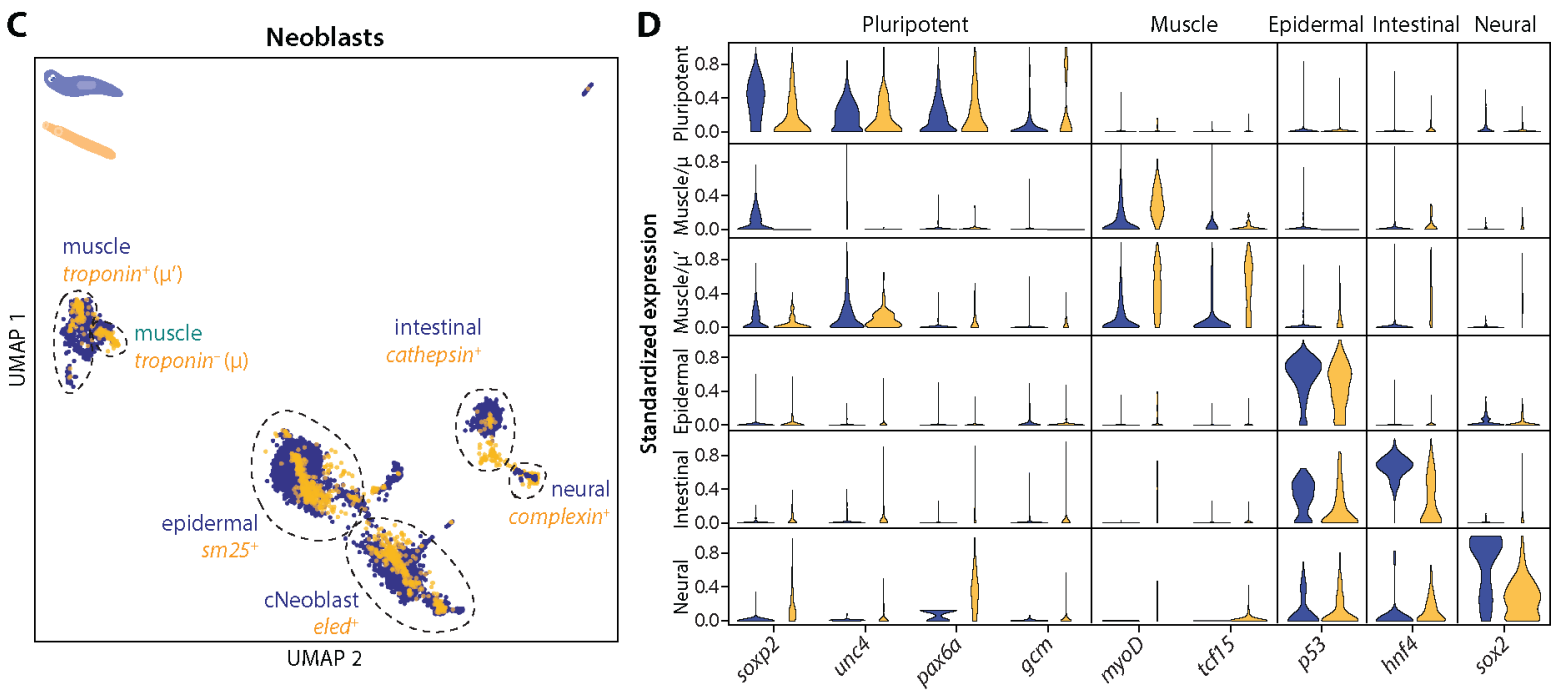

903 Figure 3: SAMap transfers cell type information from a well-annotated organism

904 (planarian S. mediterranea) to its less-studied cousin (schistosome S. mansoni)

905 and identifies parallel stem cell compartments. (A) UMAP projection of the combined

906 manifolds. Tissue type annotations are adopted from the S. mediterranea atlas (Fincher

907 et al., 2018). The schistosome atlas was collected from juvenile worms, which we found

908 to contain neoblasts with an abundance comparable to that of planarian neoblasts (Li et

909 al., 2020). (B) Overlapping expressions of selected tissue-specific TFs with expressing

910 cell types circled. (C) UMAP projection of the aligned manifolds showing planarian and

911 schistosome neoblasts, with homologous subpopulations circled. Planarian neoblast data 
912 is from (Zeng et al., 2018), and cNeoblasts correspond to the Nb2 population, which are

913 pluripotent cells that can rescue neoblast-depleted planarians in transplantation

914 experiments. (D) Distributions of conserved TF expressions in each neoblast

915 subpopulation. Expression values are k-nearest-neighbor averaged and standardized,

916 with negative values set to zero. Blue: planarian; yellow: schistosome. 


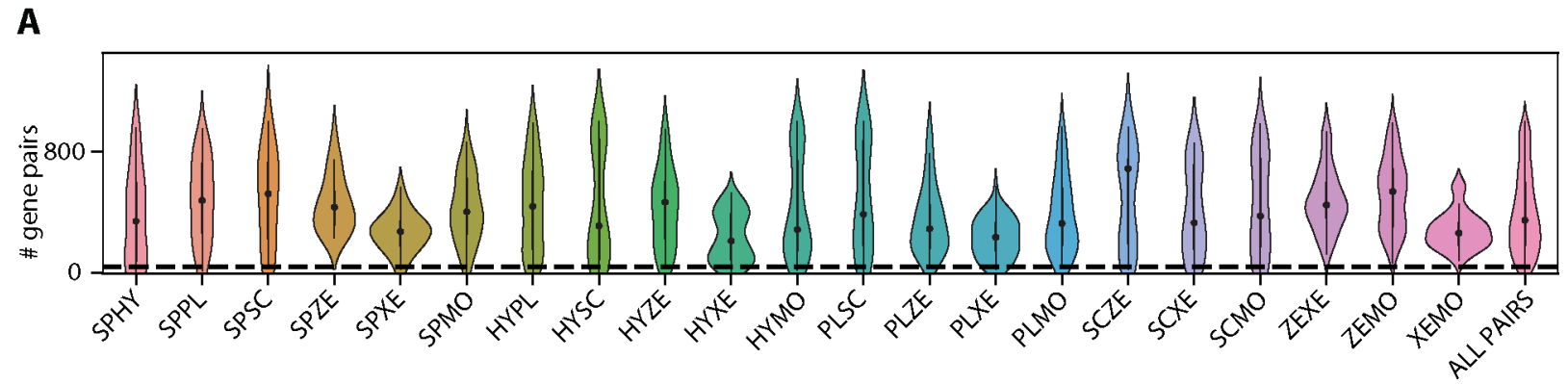

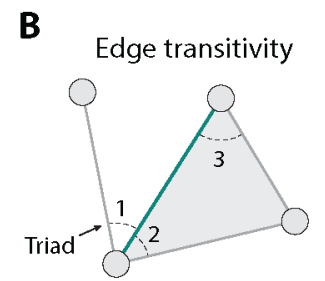

$2 / 3$ edge triads in triangle
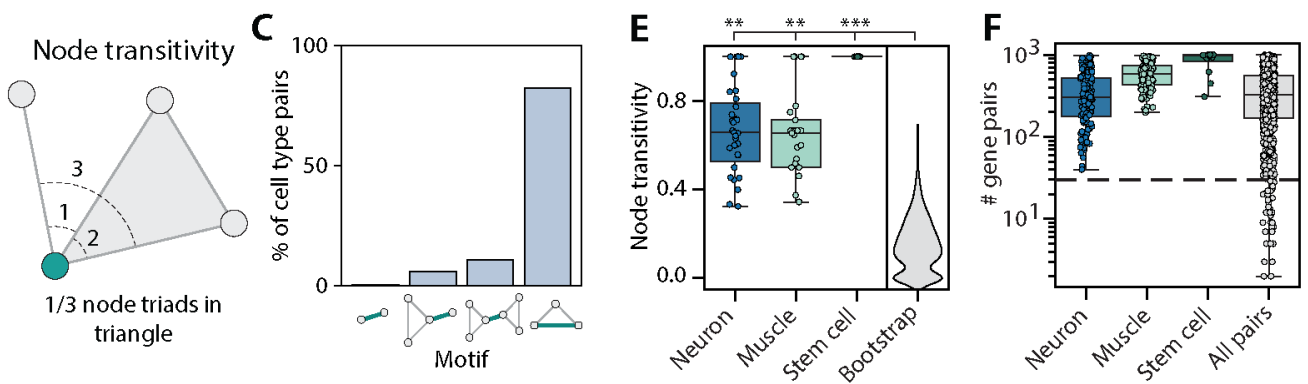

D
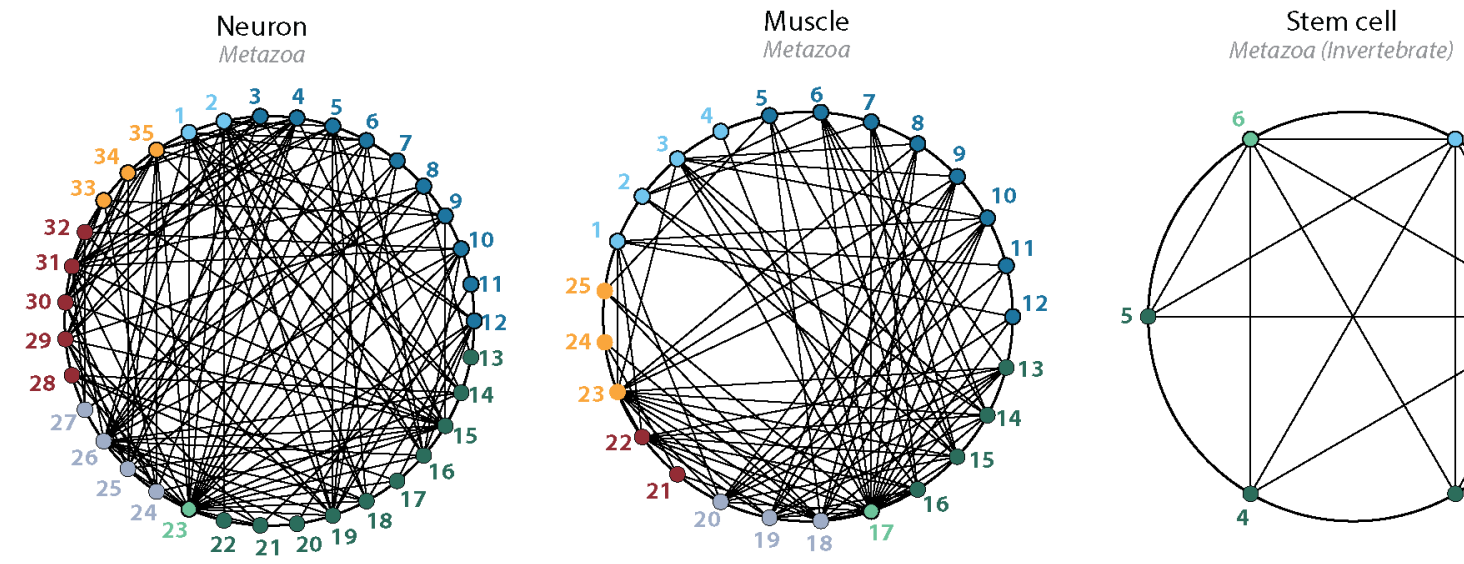

1. Apopylar cells 24-25. Brain

2. Choanocytes 26-27. Neurons

$\begin{array}{ll}\text { 3-4. Neural progenitors } & \text { 28. Neural plate } \\ \text { 5-11. Neural subpopulations } & 29-30 . \text { Brain }\end{array}$

12. Interstitial nematocyte 31-32. Neurons

13-22. Neural subpopulations 23. Spinal cord

23. Neural

20-21. Neurons

34. Rostral ineu
35. Brain

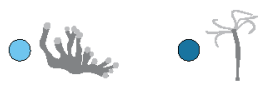

1. Myopeptidocytes

2. Basopinacocytes

3. Excurrent pinaocytes

4. Incurrent pinacocytes

5-7. Endoepithelial cells

8-12. Ectoepithelial cells

13-16. Muscle

17. Muscle

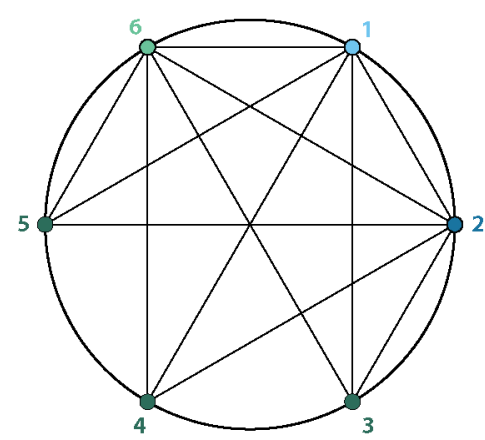

1. Archeocytes

2. Interstitial stem cells

3-5. Neoblast subpopulations

6. Neoblasts

21. Presomitic mesoderm

22. Skeletal muscle

23. Cardiomyocytes

24. Somitic mesoderm

25. Paraxial mesoderm

919 Figure 4: Mapping evolutionarily distant species identifies densely connected cell

920 type groups. (A) Violin plots showing the number of enriched gene pairs in cell type

921 mappings from all 21 pairwise mappings between the 7 species. $87 \%$ of cell type

922 mappings have greater than 40 enriched gene pairs (dotted line). Species acronyms are 
923 the same as in Figure 1A. (B) Schematic illustrating edge (left) and node (right)

924 transitivities, defined as the fraction of triads (set of three connected nodes) in closed

925 triangles. (C) The percentage of cell type pairs that are topologically equivalent to the

926 green edge in each illustrated motif. (D) Network graphs showing highly connected cell

927 type families. Each node represents a cell type, color-coded by species (detailed

928 annotations are provided in Supplementary Table 5). Mapped cell types are connected

929 with an edge. (E) Boxplot showing the median and interquartile ranges of node

930 transitivities for highly connected cell type groups. For all box plots, the whiskers denote

931 the maximum and minimum observations. The average node transitivity per group is

932 compared to a bootstrapped null transitivity distribution, generated by repeatedly

933 sampling subsets of nodes in the cell type graph and calculating their transitivities. ${ }^{*} p<$

$9345 \times 10^{-3},{ }^{* *} p<5 \times 10^{-5},{ }^{* *} p<5 \times 10^{-7}$. (F) Boxplot showing the median and interquartile

935 ranges of the number of enriched gene pairs in highly connected cell type groups. All cell

936 type connections in these groups have at least 40 enriched gene pairs (dotted line). 
A
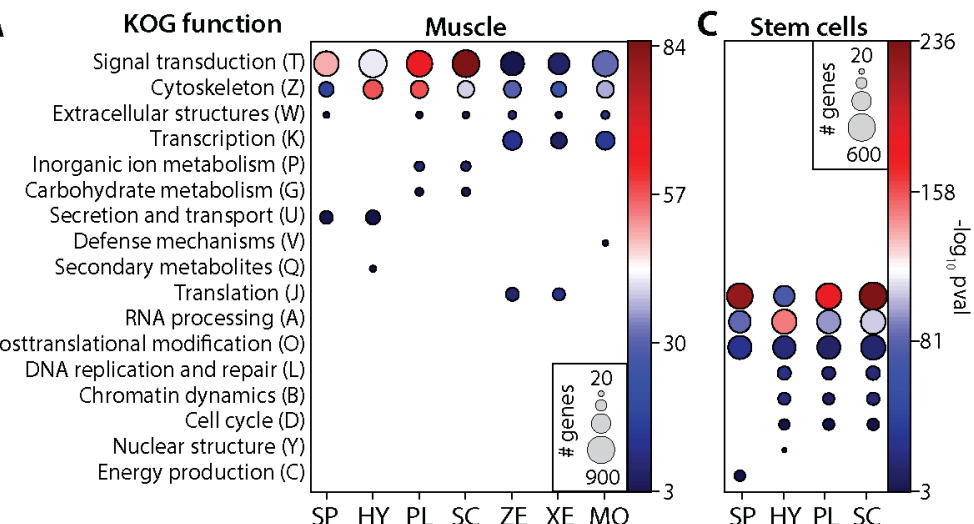

SP HY PL SC

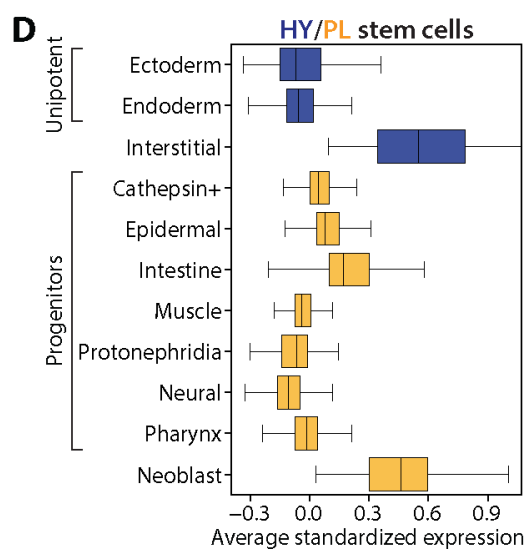

Bilateria

B

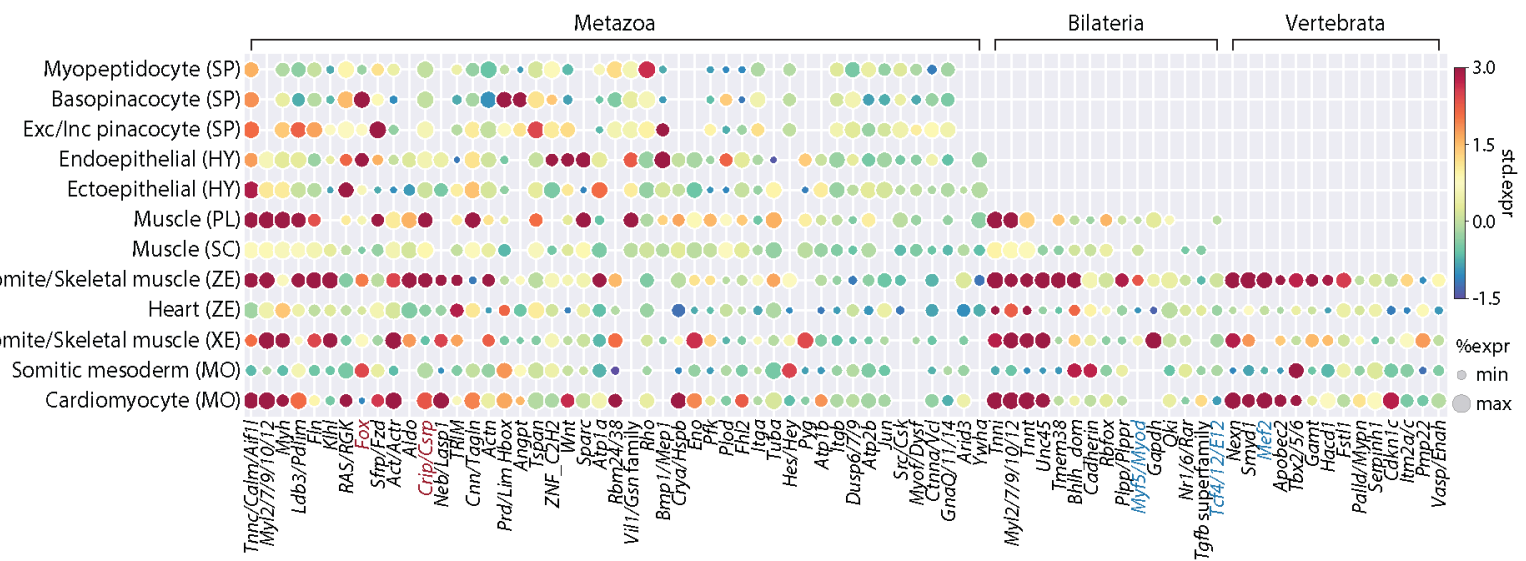

938 Figure 5: SAMap identifies muscle and stem cell transcriptional signatures

conserved across species. (A) Enrichment of KOG functional annotations calculated

940 for genes shared in contractile cell types. For each species, genes enriched in individual

941 contractile cell types are combined. (B) Expression and enrichment of conserved muscle

942 genes in contractile cell types. Color: mean standardized expression. Symbol size: the

943 fraction of cells each gene is expressed in per cell type. Homologs are grouped based on

944 overlapping eukaryotic Eggnog orthology groups. If multiple genes from a species are

945 contained within an orthology group, the gene with highest standardized expression is

946 shown. Genes in blue: core transcriptional program of bilaterian muscles; red:

947 transcription factors conserved throughout Metazoa. (C) Enrichment of KOG functional

948 annotations for genes shared by stem cell types. (D) Boxplot showing the median and 
949 interquartile ranges of the mean standardized expressions of genes in hydra and

950 planarian stem cells/progenitors that are conserved across all invertebrate species in this

951 study. Planarian progenitors: piwi+ cells that cluster with differentiated tissues in Fincher

952 et al. (Fincher et al., 2018). Neoblasts: cluster 0 in Fincher et al. (Fincher et al., 2018) that

953 does not express any tissue-specific markers. 


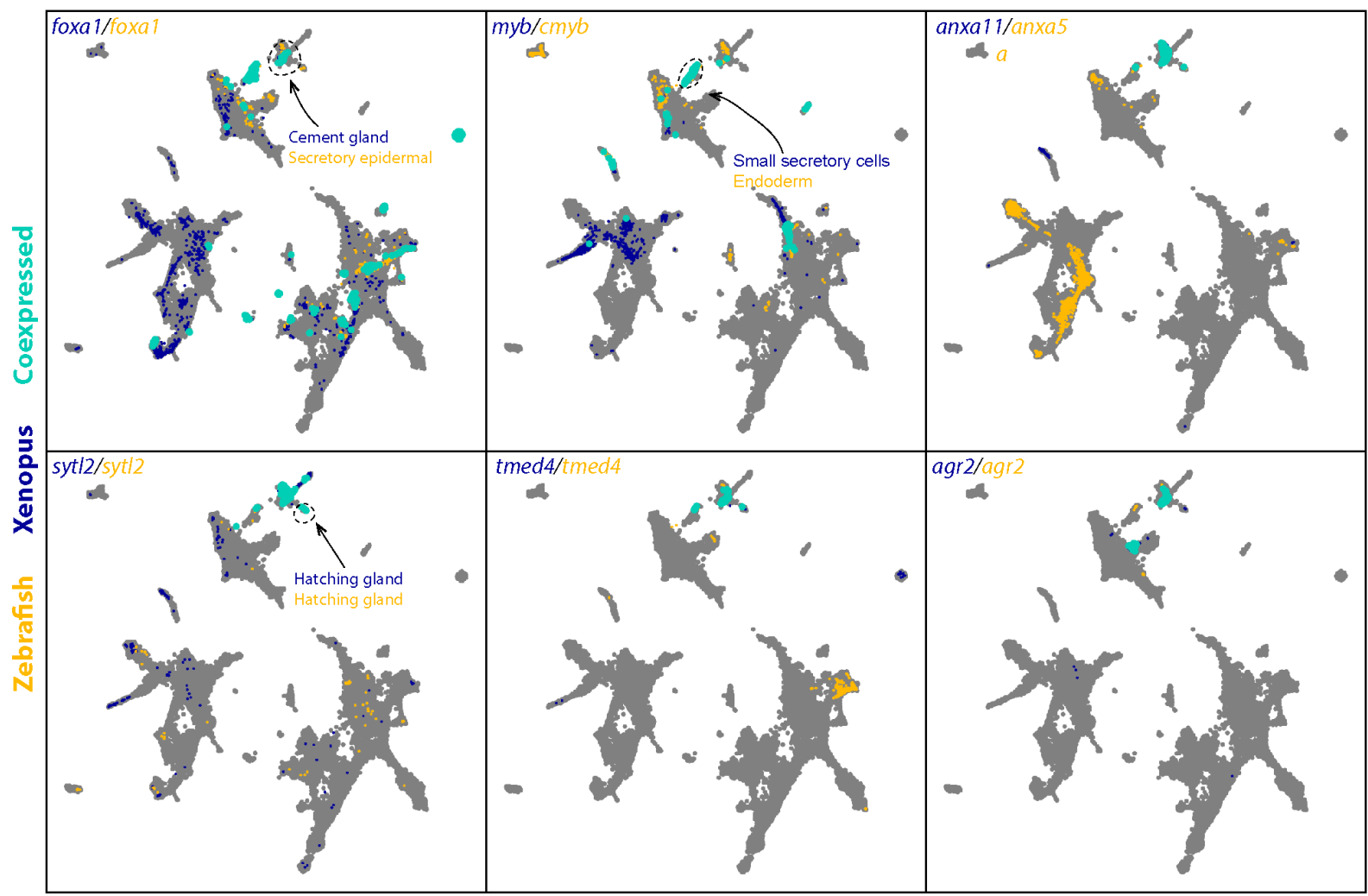

956 Figure 2 - figure supplement 1: Expression of selected genes enriched in $D$. rerio

957 and $X$. tropicalis secretory cell types. Expressions of orthologous gene pairs linked by

958 SAMap are overlaid on the combined UMAP projection. Expressing cells are color-coded

959 by species, with those connected across species colored cyan. Cells with no expression

960 are shown in gray. The mapped secretory cell types are highlighted with circles. 


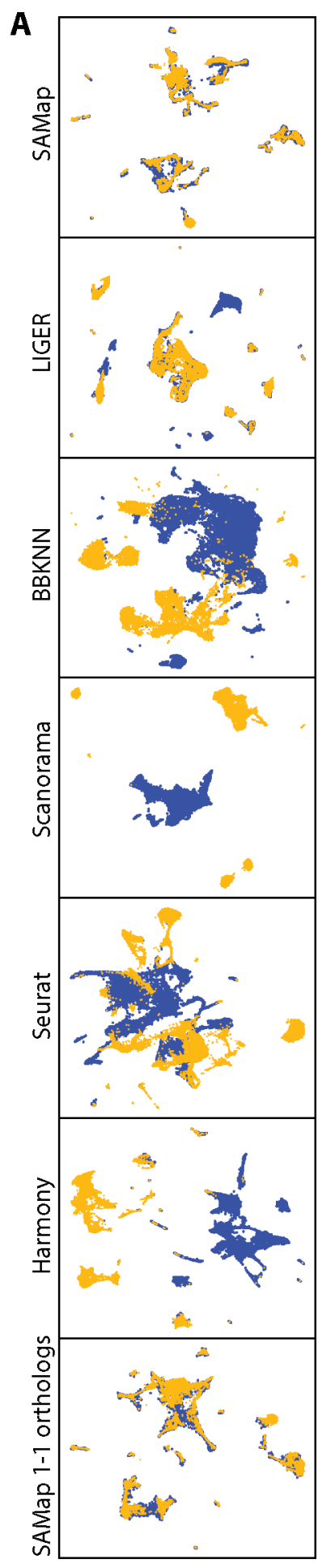

962
B
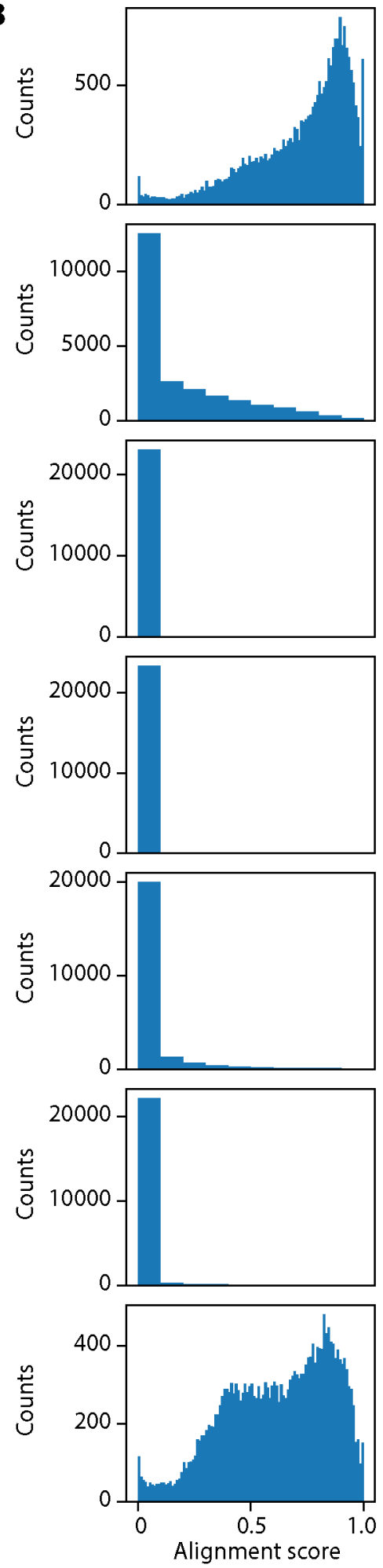

963 Figure 2 - figure supplement 2: Existing methods failed to map $D$. rerio and $X$.

964 tropicalis atlases. (A) UMAP projections of the integration results from SAMap using the 
965 full homology graph, compared to Liger, BBKNN, Scanorama, Seurat, Harmony, and

966 SAMap using 1-1 orthologs. For fair comparisons, all methods were run on the $D$. rerio

967 and $X$. torpicalis atlases subsampled to approximately 15,000 cells to satisfy

968 computational constraints of Seurat and Liger. (B) Distribution of alignment scores

969 between individual cells.

970 

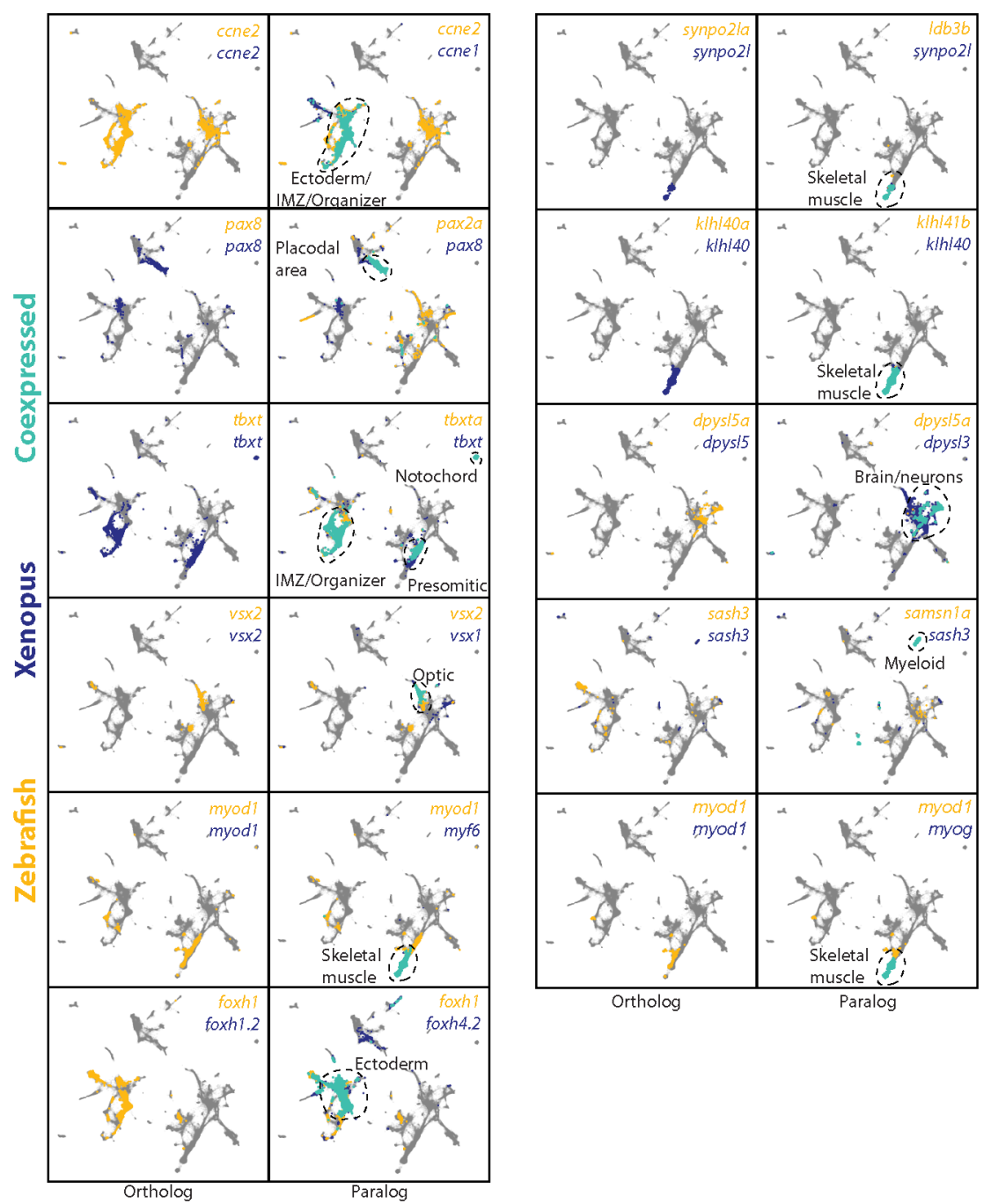

972 Figure 2 - figure supplement 3: Representative examples of paralog substitution

973 events in D. rerio and $\boldsymbol{X}$. tropicalis atlases. Expressions of orthologous and paralogous

974 gene pairs are overlaid on the combined UMAP projection. Expressing cells are color-

975 coded by species, with those that are connected across species colored in cyan. Cells

976 with no expression are shown in gray. 


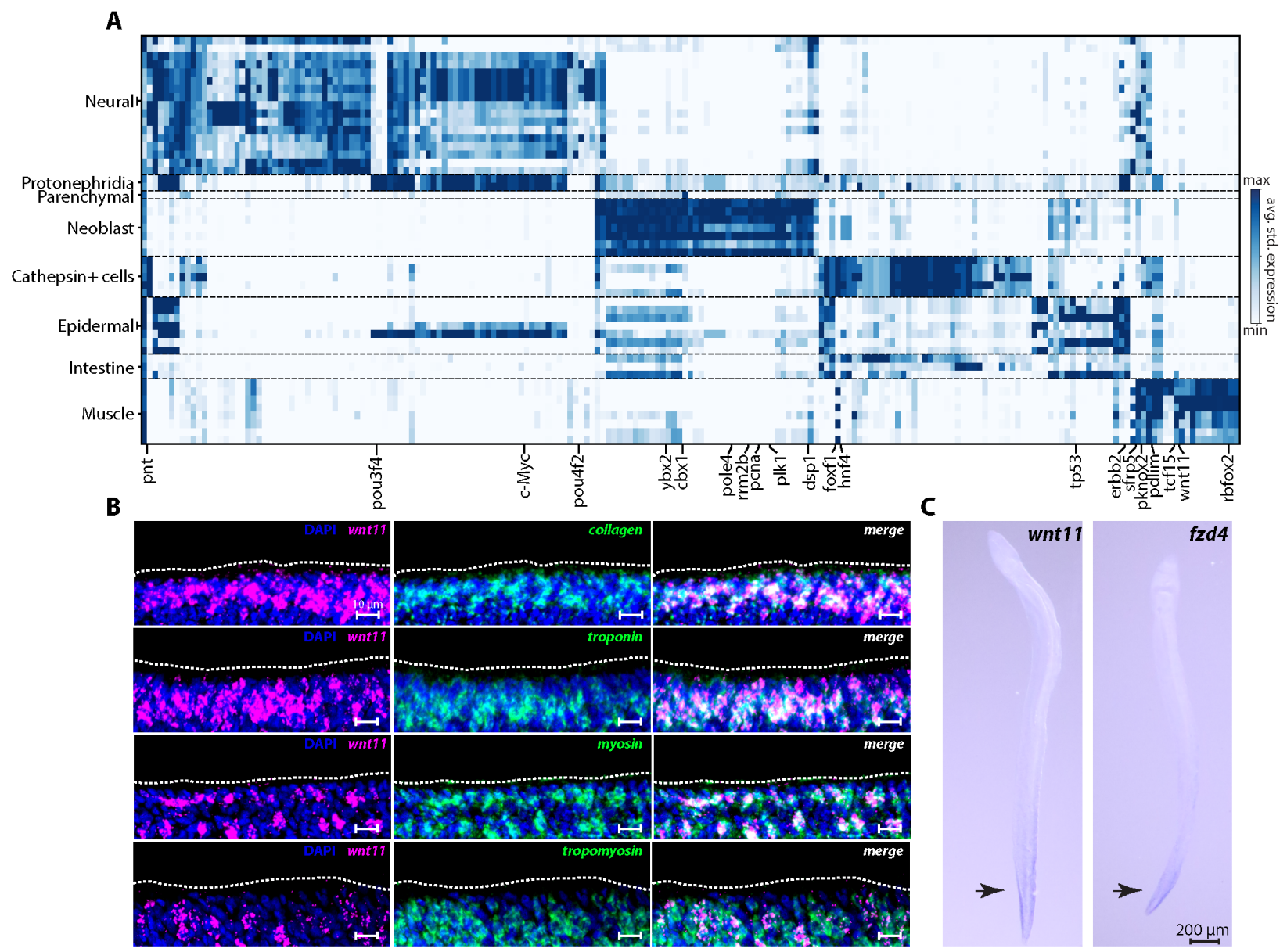

Figure 3 - figure supplement 1: SAMap-linked gene pairs that are enriched in cell

980 type pairs between S. mediterranea and S. mansoni. (A) Rows: linked cell types.

981 Schistosome cell types correspond to leiden clusters. Columns: genes linked by SAMap

982 with overlapping eukaryotic Eggnog orthology groups. We calculate the average

983 standardized expression of each gene in an orthology group for its corresponding cell

984 type in a particular pair and report the highest expression. A selected set of orthology

985 groups corresponding to transcriptional regulators are labeled. (B) Fluorescence in situ

986 hybridization shows the co-expression of wht (Smp_156540) and a panel of muscle

987 markers (collagen, troponin, myosin and tropomyosin) in S. mansoni juveniles. The body

988 wall muscles are expected to be located close to the parasite surface (dashed outline). 
989 The images are maximum intensity projections constructed from $\sim 10$ confocal slices with 990 optimal axial spacing recommended by the Zen software collected on a Zeiss LSM 800

991 confocal microscope using a $40 \times($ N.A. $=1.1$, working distance $=0.62 \mathrm{~mm})$ water992 immersion objective (LD C-Apochromat Corr M27). (C) Whole mount in situ hybridization 993 images showing that the expression of wnt and frizzled (Smp_174350) are concentrated

994 in the parasite tail (arrows) with decreasing gradients extending anteriorly. In planarian 995 muscles, Wnt genes provide the positional cues for setting up the body plan during 996 regeneration (Scimone et al., 2017; Reddien, 2018). The presence of an anterior997 posterior expression gradient of wht and frizzled in muscles of schistosome juveniles 998 suggests that they may have similar functional roles in patterning during development. 

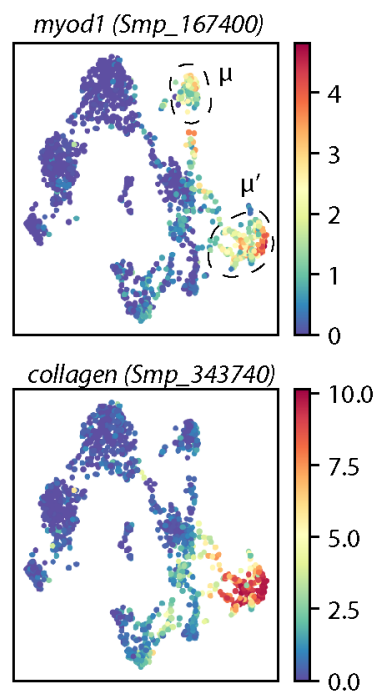
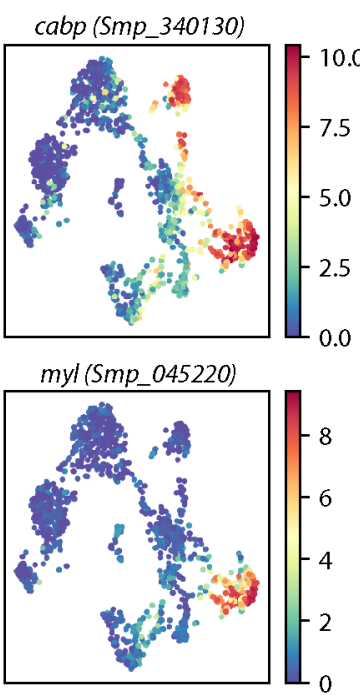

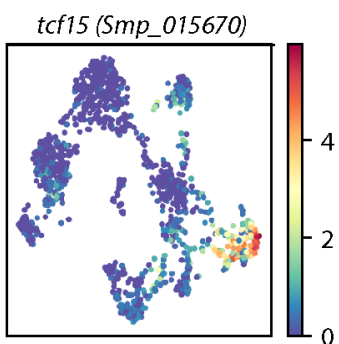

tropomyosin (Smp_031770)

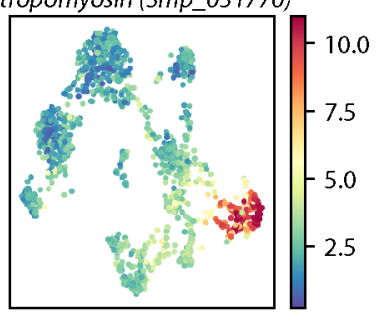

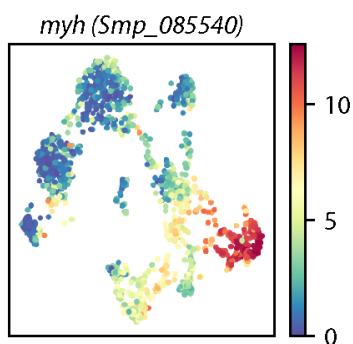

troponin (Smp_018250)

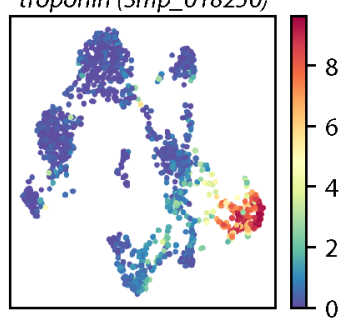

1001 Figure 3 - figure supplement 2: Schistosome neoblasts express canonical muscle markers in muscle progenitors. UMAP projections of schistosome neoblasts with gene

1003 expressions overlaid. $\mu$ and $\mu$ ' cells are circled. Colormap: expression in units of $\log _{2}(D+$ 1). For visualization, expression was smoothed via nearest-neighbor averaging using SAM. Note that myod1 and cabp are expressed in both presumptive muscle progenitor populations, whereas all other markers are enriched in $\mu^{\prime}$ cells. All genes displayed are also expressed in fully differentiated muscle tissues. 


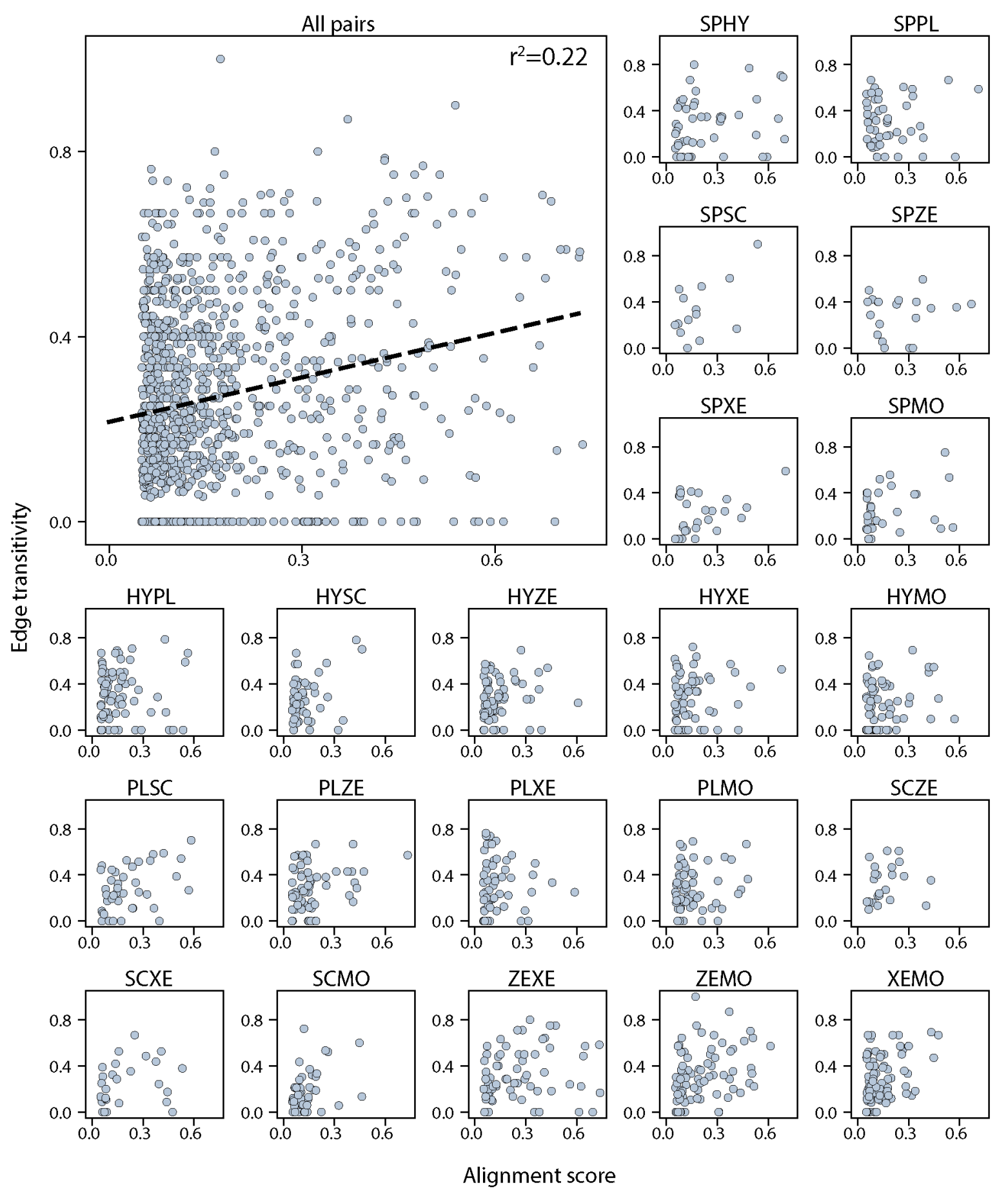

Figure 4 - figure supplement 1: Alignment scores are mostly independent of edge

1011 transitivity. Top left: alignment scores and edge transitivity for all cell type pairs in the

1012 connectivity graph including the 7 species. Dotted line: the linear best fit, with the Pearson 
1013 correlation coefficient reported at the top. Alignment scores and edge transitivity for 1014 individual species pairs are shown in the remaining subplots.

1015 


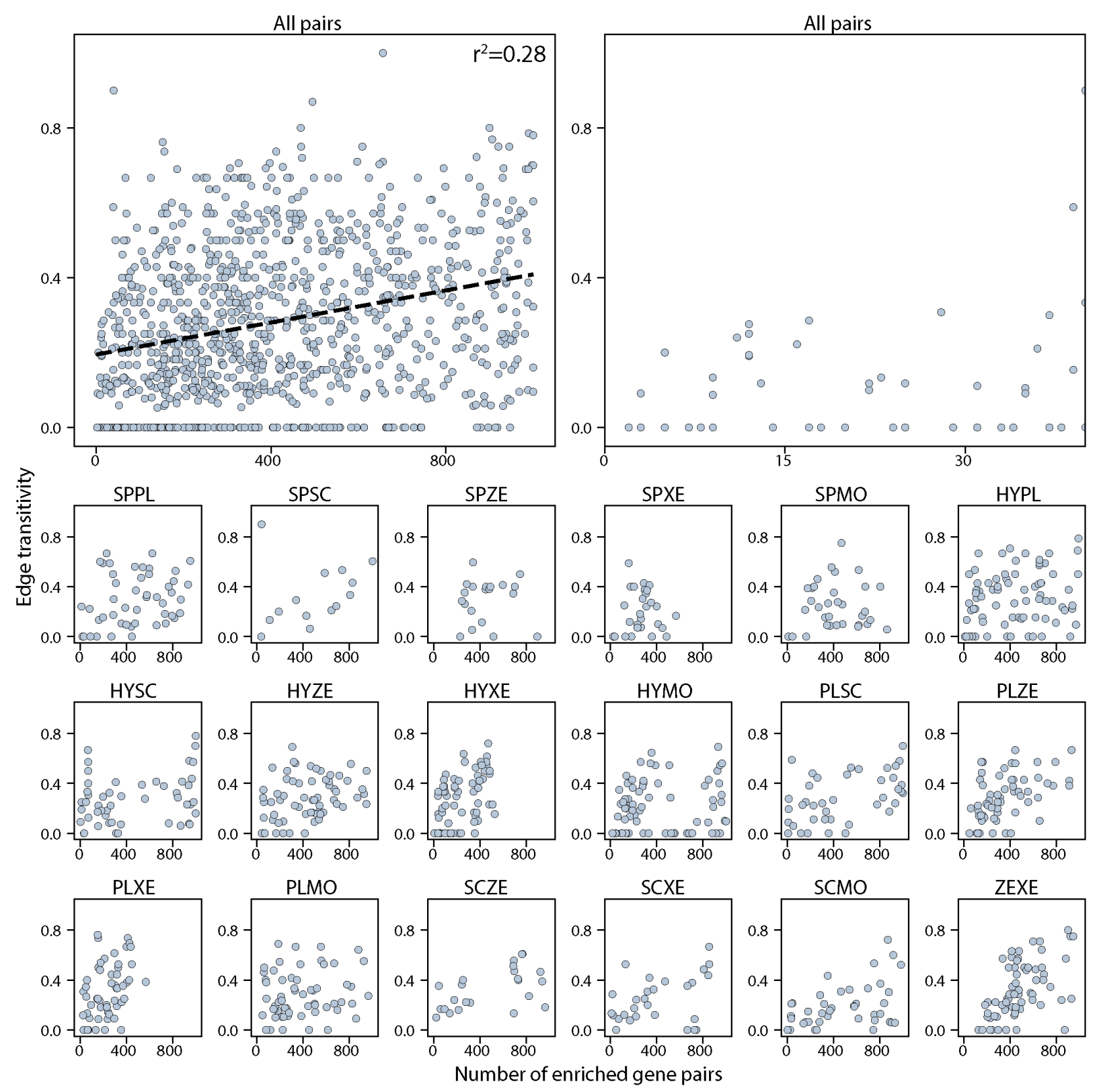

1017 Figure 4 - figure supplement 2: Number of enriched gene pairs are mostly

1018 independent of edge transitivity. Top left: The edge transitivity is plotted against the

1019 number of enriched gene pairs for all cell type pairs in the connectivity graph. Dotted line:

1020 the linear best fit, with the Pearson correlation coefficient reported at the top. Top right:

1021 magnified view of the mapped cell type pairs supported by small numbers of gene pairs 
$1022(<40)$ to show those edges have low transitivity scores $(<0.4)$. The sublots below show 1023 the number of enriched gene pairs and edge transitivity for individual species pairs.

1024 
bioRxiv preprint doi: https://doi.org/10.1101/2020.09.28.317784; this version posted March 19, 2021. The copyright holder for this preprint (which was not certified by peer review) is the author/funder, who has granted bioRxiv a license to display the preprint in perpetuity. It is made available under aCC-BY-NC-ND 4.0 International license.

A

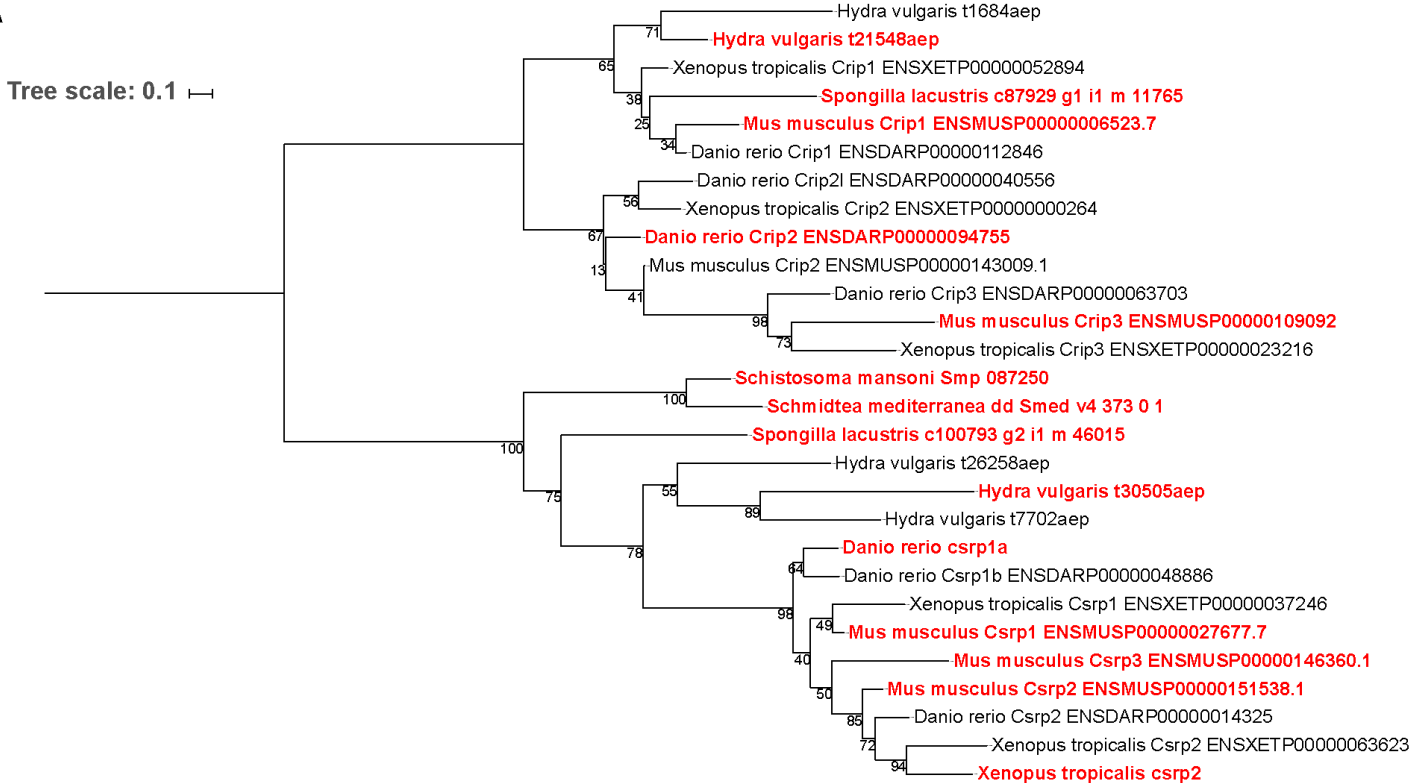

\section{B}

Tree scale: 0.1

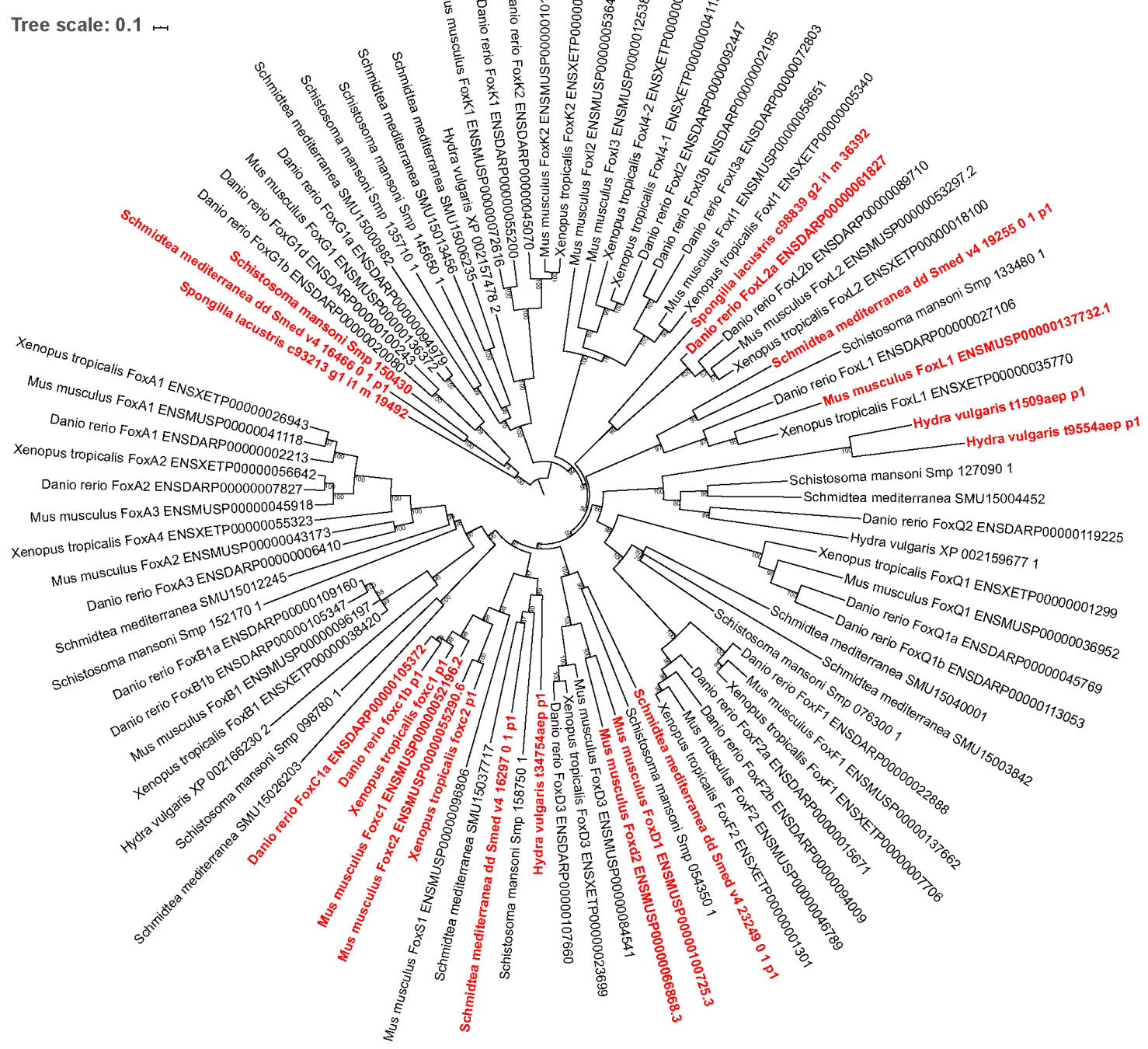


1026 Figure 5 - figure supplement 1: Phylogenetic reconstruction of animal contractile

1027 cell transcriptional regulators. Trees depict Csrp/Crip (A) and Fox group I (B) gene

1028 families. Genes labelled red are enriched in at least one contractile gene pair identified

1029 via SAMap. Support values indicate bootstrap support from 1,000 nonparametric (Csrp)

1030 or ultrafast (Fox) bootstrap replicates. Besides these two transcriptional regulators,

1031 contractile cells in all seven species were found to be also enriched for transcription

1032 factors from the $\mathrm{C} 2 \mathrm{H} 2$ Zinc Finger, Lim Homeobox, and Paired Homeobox families,

1033 although in different cell types we found enrichment of a number of distinct orthologs.

1034 Whether this reflects an ancestral role for these transcription factor families in regulating

1035 contractility or their independent evolution will require additional taxonomic sampling and

1036 broader coverage of muscle cell diversity to resolve. 


\section{Supplementary table captions}

Supplementary Table 1: Cell atlas metadata and cell annotations. Metadata includes

1040 the number of cells, number of transcripts in the transcriptome, median number of

1041 transcripts detected per cell, the reference transcriptome used in this study, database

1042 through which the transcriptomes are provided, technology used for constructing the cell

1043 atlases, atlas data accessions, processing notes, and references. Leiden clusters and

1044 cell type annotations are reported for cells in each atlas. The Zebrafish and Xenopus

1045 tables include both the original cell type annotations and those used in this study. D. rerio,

$1046 X$. tropicalis, and mouse annotations include developmental stages.

Supplementary Table 2: Cell type annotations for the zebrafish-Xenopus mapping.

1049 Correspondence between the cell type annotations provided in the original study (Briggs 1050 et al., 2018; Wagner et al., 2018) and corresponding annotations used in this study is 1051 provided for both $D$. rerio and $X$. tropicalis atlases.

1052

Supplementary Table 3: Identified paralogs with greater expression similarity than

1054 orthologs in the zebrafish-Xenopus mapping. Each row contains a pair of vertebrate-

1055 orthologous genes and a corresponding pair of eukaryotic paralogs with higher correlation

1056 in expression compared to the orthologs, the expression correlations for ortholog and 1057 paralog pairs, the difference between their correlations, and whether the paralogs are 1058 considered as a paralog substitution (defined as when the substituted ortholog is either 
1059 absent or lowly-expressed with no cell-type specificity). Highlighted rows are shown in

1060 Figure 2E and Figure 2 - figure supplement 3.

Supplementary Table 4: Genes enriched in contractile cell types and invertebrate

stem cells highlighted in Figure 4D. The IDs of the genes enriched in the contractile

1064 and invertebrate stem cell types are provided along with the IDs of the Eggnog orthology

1065 groups to which they belong. In cases where multiple genes from a species belonging to

1066 the same orthology group are enriched, the most differentially expressed gene is shown.

1067 The descriptions in the stem cell table are orthology annotations associated with the

1068 Spongilla genes provided in the original study (Musser et al., 2019).

Supplementary Table 5: Cell types in the cell type families shown in Figure 4D. For

1071 the schistosome cell types, we annotated two neural clusters, both of which express the

1072 neural marker complexin (Li et al., 2020). One of the clusters expresses the antigen

1073 SmKK7, so we label the clusters "Neural" and "Neural_KK7", respectively. The "Muscle"

1074 population contains non-neoblast cells expressing troponin. The "Tegument_prog" and

1075 "Tegument" populations consist of cells expressing tegument progenitor and

1076 differentiated marker genes, respectively, as reported in a previous study (Wendt et al., 1077 2018). 